\title{
LPCAT1 regulates surfactant phospholipid synthesis and is required for transitioning to air breathing in mice
}

\author{
James P. Bridges, ${ }^{1}$ Machiko Ikegami, ${ }^{1}$ Lauren L. Brilli, ${ }^{1}$ Xueni Chen, ${ }^{2}$ \\ Robert J. Mason, ${ }^{2}$ and John M. Shannon'1 \\ ${ }^{1}$ Division of Pulmonary Biology, Cincinnati Children's Hospital Medical Center, Cincinnati, Ohio, USA. ²Department of Medicine, \\ National Jewish Medical and Research Center, Denver, Colorado, USA.
}

\begin{abstract}
Respiratory distress syndrome (RDS), which is the leading cause of death in premature infants, is caused by surfactant deficiency. The most critical and abundant phospholipid in pulmonary surfactant is saturated phosphatidylcholine (SatPC), which is synthesized in alveolar type II cells de novo or by the deacylation-reacylation of existing phosphatidylcholine species. We recently cloned and partially characterized a mouse enzyme with characteristics of a lung lysophosphatidylcholine acyltransferase (LPCAT1) that we predicted would be involved in surfactant synthesis. Here, we describe our studies investigating whether LPCAT1 is required for pulmonary surfactant homeostasis. To address this issue, we generated mice bearing a hypomorphic allele of $L p c a t 1$ (referred to herein as $L p c a t 1^{G T / G T}$ mice) using a genetrap strategy. Newborn $L p c a t 1^{G T / G T}$ mice showed varying perinatal mortality from respiratory failure, with affected animals demonstrating hallmarks of respiratory distress such as atelectasis and hyaline membranes. Lpcat $1 \mathrm{mRNA}$ levels were reduced in newborn $L p c a t 1{ }^{G T / G T}$ mice and directly correlated with SatPC content, LPCAT1 activity, and survival. Surfactant isolated from dead $L p c a t 1^{G T / G T}$ mice failed to reduce minimum surface tension to wild-type levels. Collectively, these data demonstrate that full LPCAT1 activity is required to achieve the levels of SatPC essential for the transition to air breathing.
\end{abstract}

\section{Introduction}

Maintenance of alveolar patency is critical for gas exchange. Pulmonary surfactant is a lipoprotein complex that coats the luminal surface of alveoli to reduce surface tension to near $0 \mathrm{mN} / \mathrm{m}$ at the air-liquid interface and prevent alveolar collapse at end-expiration. Deficiencies and/or dysfunction of the surfactant system are known to contribute to the pathogenesis of several pulmonary diseases, including neonatal respiratory distress syndrome (RDS) (1), acute lung injury (ALI) (1-5), and interstitial lung disease in both children and adults (6). While exogenous surfactant therapy has substantially reduced morbidity and mortality in the premature infant population, RDS and ALI remain significant health problems.

Surfactant is synthesized, stored, and secreted by alveolar type II cells and is primarily composed of phospholipids, which constitute $80 \%-85 \%$ of the total mass. The remaining components of surfactant include neutral lipids (5\%-10\%) and proteins $(10 \%)$ including SFTPA, SFTPB, SFTPC, and SFTPD $(7,8)$. Phosphatidylcholine (PC), the most abundant phospholipid species in surfactant, constitutes $80 \%$ of the total phospholipids. The majority of PC in surfactant contains 2 saturated fatty acid chains (SatPC), a unique property that distinguishes it from PC species found in cell membranes. Most of the SatPC exists as dipalmitoyl-PC (DPPC; 16:0/16:0), with the remainder as palmitoyl-myristoyl-PC (PMPC; 16:0/14:0) (9). SatPC is the phospholipid most responsible for the surface tension-lowering properties of surfactant, a function that is directly attributable to its ability to be tightly packed during cyclical compression and expansion $(10,11)$.

Synthesis of SatPC occurs either by direct de novo biosynthesis or by the remodeling of existing monosaturated PC species.

Conflict of interest: The authors have declared that no conflict of interest exists. Citation for this article: J Clin Invest. 2010;120(5):1736-1748. doi:10.1172/JCI38061.
In vitro studies on surfactant synthesis in lung microsomes and isolated type II cells have estimated that $55 \%-75 \%$ of the SatPC is synthesized by the remodeling pathway $(12,13)$. Formation of SatPC by the remodeling pathway occurs in 2 concerted reactions: deacylation of monosaturated PC by a phospholipase $\mathrm{A}_{2}\left(\mathrm{PLA}_{2}\right)$, followed by reacylation of the resultant 1-palmitoyl-2-lysophosphatidylcholine with palmitoyl-CoA by a lysophosphatidylcholine (lysoPC) acyltransferase. While the importance of the remodeling pathway to SatPC production has been known for many years (14-17), little is known regarding the regulation of this pathway, largely due to an inability to clone the enzymes involved.

Our laboratory and an independent group have recently cloned and partially characterized a lung lysoPC acyltransferase (LPCAT1) (18, 19). In the mouse lung, LPCAT1 expression is restricted to alveolar type II cells and is developmentally regulated, with peak expression at E18.5. LPCAT1 shows a high affinity for palmitoyl-CoA as an acyl donor and preferentially acylates lysoPC over other lysophospholipid substrates $(18,19)$. Furthermore, LPCAT1 expression is increased by glucocorticoids and keratinocyte growth factor (KGF) in vitro (18), both of which are known to stimulate phospholipid biogenesis in alveolar type II cells (20-22). Because the temporal-spatial expression pattern and substrate specificity of LPCAT1 are consistent with reported lysoPC acyltransferase activity in the lung, we hypothesized that LPCAT1 is involved in surfactant SatPC synthesis.

In the present study, we confirm and extend our previous in vitro data by demonstrating that LPCAT1 is an ER-resident type II transmembrane protein with a motif critical for catalytic activity localized to the ER lumen. Furthermore, suppression of LPCAT1 in isolated type II cells results in decreased SatPC production, whereas overexpression of LPCAT1 is sufficient to increase SatPC synthesis and secretion in MLE15 cells. Finally, we demonstrate that neonatal mice homozygous for a hypomorphic allele of Lpcat1 

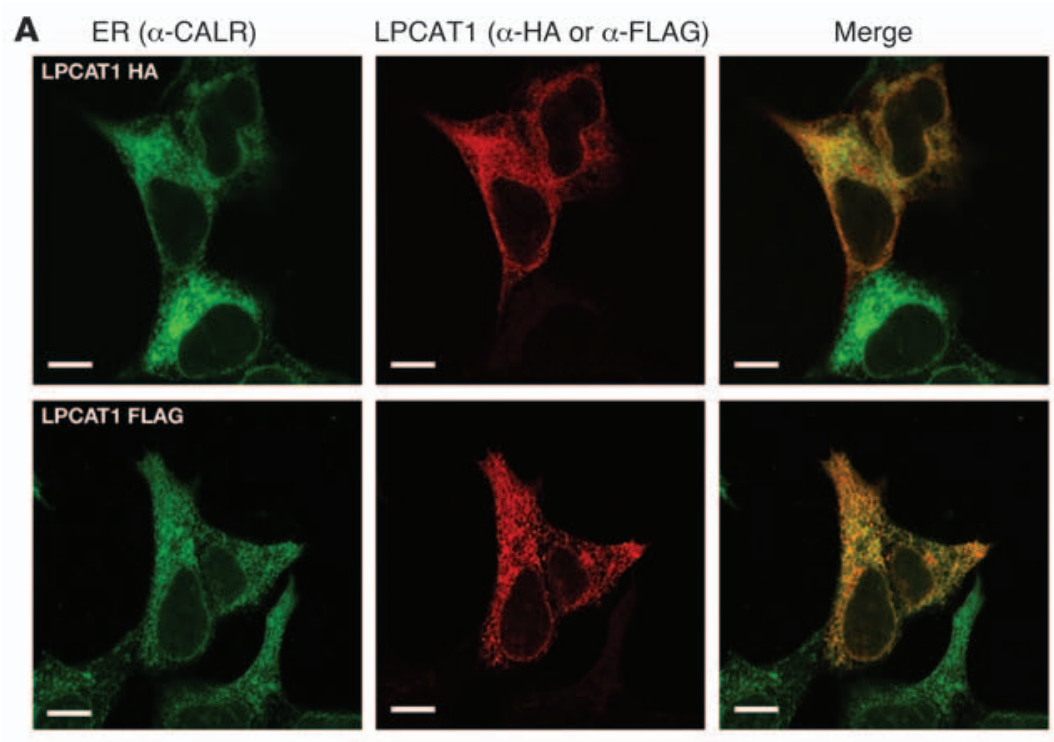

C

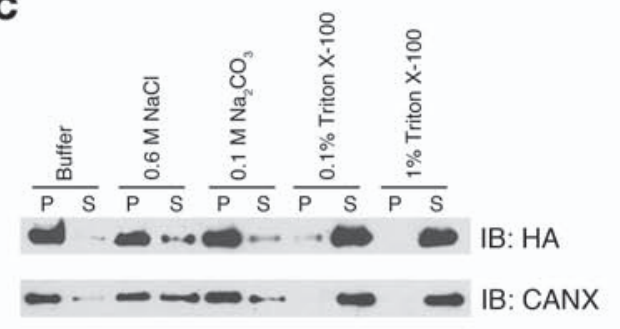

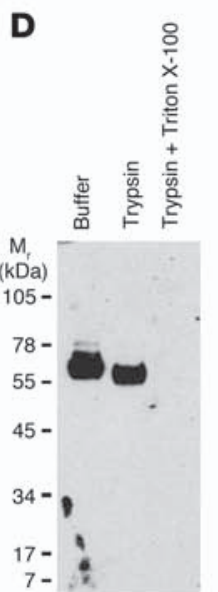

IB: HA

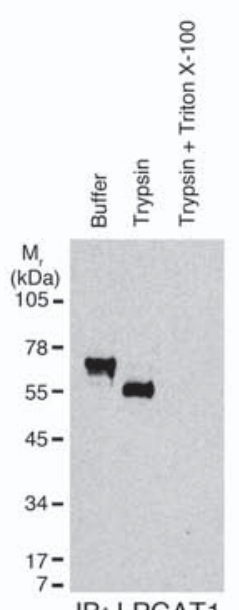

IB: LPCAT1
B
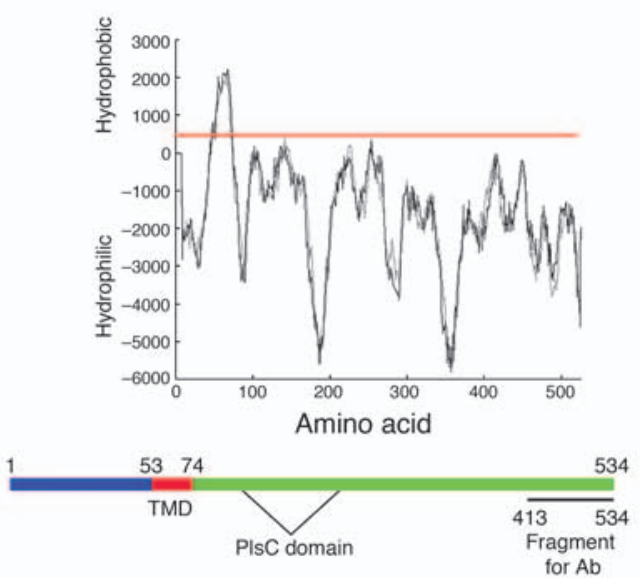

Type II orientation

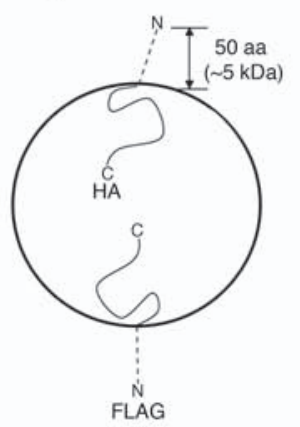

Figure 1

LPCAT1 is a type II transmembrane protein localized to the ER. (A) HEK293 cells transfected with LPCAT-HA or LPCAT-FLAG were costained with $\alpha-H A$ and $\alpha$-CALR or $\alpha$-FLAG and $\alpha$-CALR. Note the localization of LPCAT-HA and LPCAT-FLAG proteins to the ER. Scale bars: $10 \mu m$. (B) Hydropathy plot of mouse LPCAT1 protein as predicted from the TMpred algorithm. Scores greater than 500 (red line) are considered hydrophobic. A single TMD is predicted, encompassing amino acids 53-74. Bottom panel: Diagram depicts the position of the TMD of LPCAT1 protein relative to the acyltransferase (PIsC) domain. (C) Membrane fractionation assay on HEK293 cells expressing LPCAT1-HA. Pellet (P) and supernatant (S) fractions were isolated after incubation of the membranes in the listed additives and subjected to immunoblot analysis with $\alpha-\mathrm{HA}$ antibody. Blots were stripped and reprobed with an antibody against endogenous CANX, an ER-resident transmembrane protein. (D) Trypsin protection assay. Microsomes isolated from HEK293 cells transiently expressing LPCAT-HA or LPCAT-FLAG were incubated with buffer only, trypsin, or trypsin plus Triton X-100 and subjected to immunoblot analysis. Note the approximate 5-kDa shift of the LPCAT-HA protein in the presence of trypsin (IB: HA and IB: LPCAT1) and failure to detect LPCAT-FLAG in the presence of trypsin (IB: FLAG), indicating a type II orientation. A model depicting LPCAT1 in a type II orientation within a microsome is shown on the right.

show a varying mortality from respiratory distress, with Lpcat1 mRNA and acyltransferase activity correlating directly with SatPC content, surfactant function, and survival. Taken together, these data demonstrate that LPCAT1 is required for the transition to air breathing and underscore the importance of the remodeling pathway for SatPC synthesis in vivo.

\section{Results}

LPCAT1 is a type II transmembrane protein localized to the ER. For immunohistochemical and biochemical analyses of LPCAT1, we generated 2 expression constructs: LPCAT-HA with an in-frame $\mathrm{HA}$ tag at the $\mathrm{C}$ terminus and LPCAT-FLAG with an in-frame FLAG tag at the $\mathrm{N}$ terminus. Because the ER is a major site of phospholipid synthesis, we predicted that LPCAT1 would localize to this compartment. Both tagged LPCAT1 proteins colocalized with CALR, an ER-resident protein, in transfected HEK293 cells, confirming and extending results from a previous study (19) (Figure 1A). In that same study, however, LPCAT1 was reported to contain 3 putative transmembrane domains (TMDs) (19). Using the TMpred algorithm (see Methods for URL) to identify mem- 


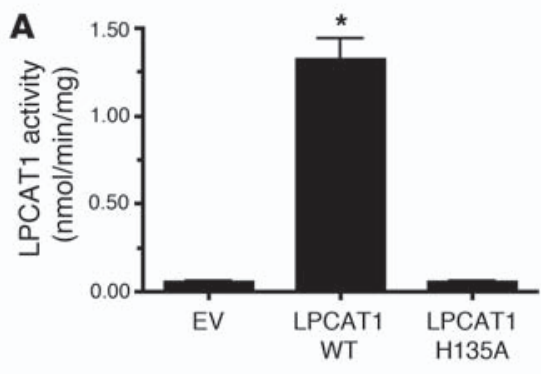

B

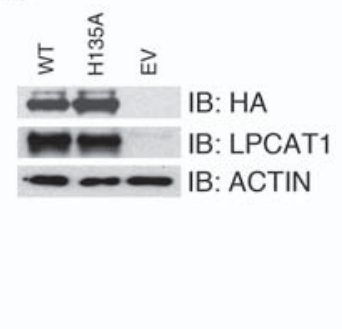

C $\operatorname{ER}(\alpha-\mathrm{CALR})$
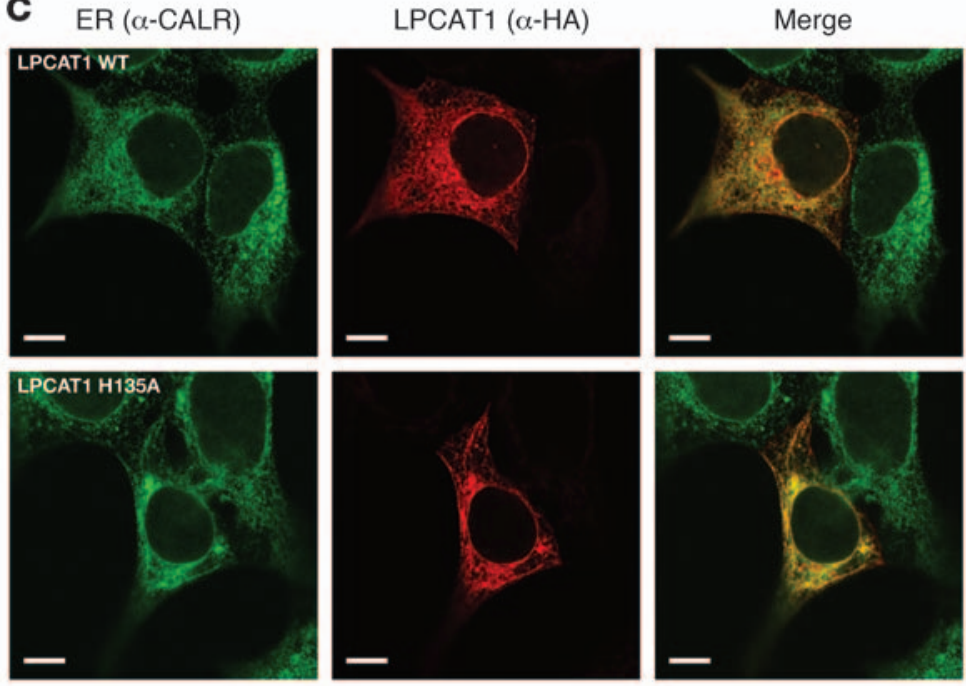

D

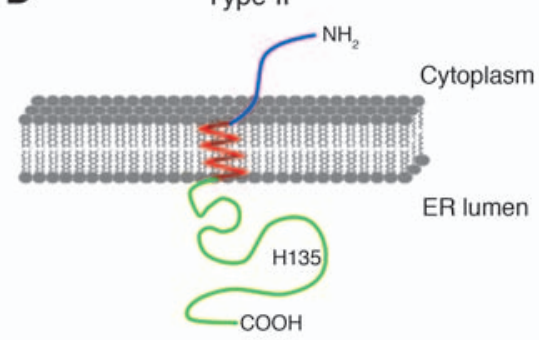

\section{Figure 2}

His135 is critical for LPCAT1 activity. (A) Acyltransferase activity assays were conducted by incubating $150 \mu \mathrm{M}$ lysoPC and $25 \mu \mathrm{M} 1{ }^{14} \mathrm{C}$-palmitoyl-CoA with $20 \mu \mathrm{g}$ of lysates from cells transfected with empty vector $(E V)$, wild-type LPCAT1 (LPCAT1 WT), or LPCAT1 with an alanine substitution for histidine at amino acid position 135 (LPCAT1 $\mathrm{H} 135 \mathrm{~A}$ ). The complete abrogation of acyltransferase activity by the substitution of alanine for histidine identifies it as a critical residue. Data represent activities from 3 independent experiments with each group assayed in triplicate. ${ }^{*} P<0.001$ versus EV. (B) Immunoblot analysis of whole cell lysates from A with $\alpha$-HA and $\alpha$-LPCAT1 antibodies. Note similar levels of expression of WT and H135A LPCAT1. (C) Subcellular localization of LPCAT WT and LPCAT H135A to ER in HEK293 cells. Merged images demonstrate colocalization (yellow) of HA epitope (red) and an ER marker, CALR (green). Scale bars: $10 \mu \mathrm{m}$. (D) Diagram depicting type II orientation of LPCAT1 in ER membrane with amino terminus (blue) in cytosol, a single-pass TMD (red) spanning the lipid bilayer, and carboxyl terminus (green) in ER lumen. Note the localization of His135 (H135) in ER lumen.

topology of LPCAT1 in the ER membrane using trypsin protection assays on membrane fractions isolated from LPCAT-HA- or LPCAT-FLAG-transfected cells. Treatment of microsomes containing LPCAT-HA with trypsin resulted in a $5-\mathrm{kDa}$ shift in anti-HA and anti-LPCAT1 immunoblots, consistent with digestion of the $50 \mathrm{~N}$-terminal amino acids of LPCAT-HA (Figure 1D). Conversely, LPCAT-FLAG was undetectable in anti-FLAG immunoblots of trypsin-treated microsomes, demonstrating susceptibility of the N-terminal FLAG epitope to enzymatic digestion. Collectively, these results demonstrate that LPCAT 1 contains a single TMD and is oriented in a type II fashion within the ER membrane.

Histidine 135 is critical for the acyltransferase activity of LPCAT1. Protein sequence analyses of glycerolipid acyltransferases from bacteria, plants, and animals have shown that this family of enzymes possesses a highly conserved motif consisting of 2 invariant residues, histidine and aspartic acid, separated by 4 nonconserved

brane-spanning domains, we predicted LPCAT1 protein to contain a single putative TMD located between amino acids 53 and 74 (Figure 1B). Identical results were obtained using 2 separate algorithms: SOSUI and Kyte-Doolittle hydropathy plot (data not shown; see Methods for URLs).

To determine whether LPCAT1 possessed the biochemical properties of a transmembrane protein, we performed membrane fractionation assays on LPCAT1-transfected cells. While LPCAT-HA remained primarily membrane associated in the presence of high $\mathrm{NaCl}$ or high $\mathrm{pH}$, the protein partitioned to the soluble fraction in the presence of detergent, properties identical to those of the endogenous transmembrane protein CANX (Figure 1C). Proteins containing a single TMD adopt one of two orientations in the ER membrane: type II, with the $\mathrm{N}$ terminus in the cytosol and the $\mathrm{C}$ terminus in the ER lumen; and type III, with the $\mathrm{N}$ terminus in the ER lumen and the $\mathrm{C}$ terminus in the cytosol. Because the acyltransferase domain $(\mathrm{PlsC}$ ) is C-terminal to the TMD (Figure 1B), we predicted LPCAT1 to be in a type II orientation, thereby placing the catalytic domain in the lumen of the ER. We assessed the residues in an $\mathrm{HX}_{4} \mathrm{D}$ configuration (23). Substitution of alanine for the conserved histidine in this motif ablated acyltransferase activity of PlsB, the bacterial sn-glycerol-3-phosphate acyltransferase (23). Alignment of the primary amino acid sequences of LPCAT1 from mouse, rat, and human revealed an $\mathrm{HX}_{4} \mathrm{D}$ motif consisting of HSSYFD at amino acids $135-140$. To determine whether histidine 135 (His135) is critical for the acyltransferase activity of LPCAT1, we performed acyltransferase activity assays using an LPCAT1 expression construct with an alanine substitution for histidine at position 135 (H135A). Substitution of an alanine for His 135 completely ablated the acyltransferase activity of LPCAT-HA (Figure 2A) without affecting protein expression levels (Figure 2B) or ER localization (Figure 2C). These results confirm those previously reported by Harayama et al. (24). Similar assays were performed with a construct harboring a D140A mutation to determine the importance of the conserved aspartic acid residue; mutation of D140, however, severely destabilized the protein, precluding interpretation of the functional assays (data not shown). These functional data, combined with the biochemical data from Figure 1, 
A

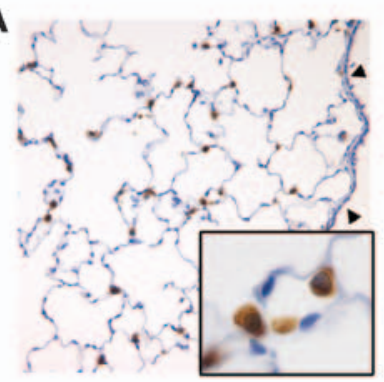

B

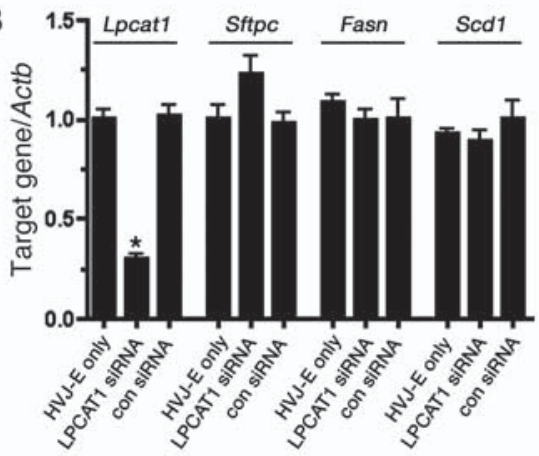

C
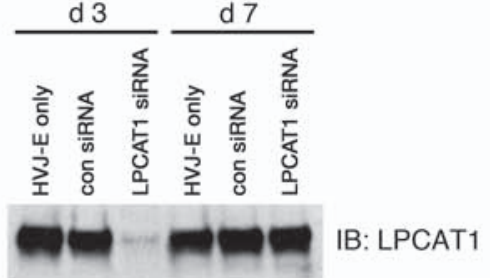

IB: ACTIN
D

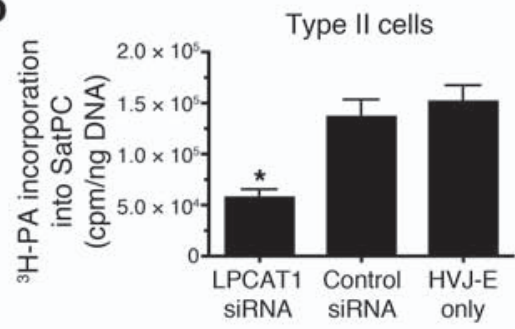

E

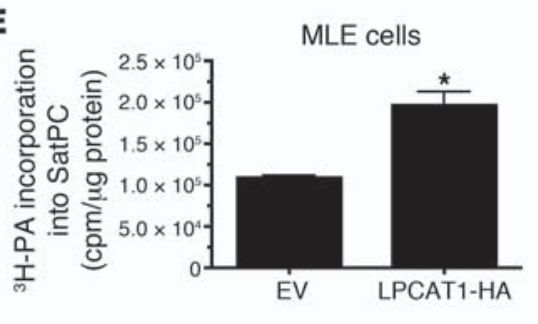

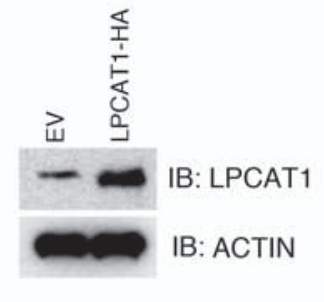

Figure 3

LPCAT1 is required for SatPC synthesis in alveolar type II cells. (A) Immunohistochemistry of adult mouse lung with $\alpha$-LPCAT1 antibody demonstrating restricted expression in alveolar type II cells. Note robust staining in alveolar type II cells (inset) and absence of signal in the proximal epithelium (arrowheads). Original magnification, $\times 40$; inset magnification, $\times 100$. (B) qPCR analysis of Lpcat1, Sttpc, Fasn, and Scd1 mRNA in primary mouse type II cells after treatment with LPCAT1 siRNA or a scrambled control siRNA (con siRNA) for 48 hours. Gene expression was normalized to Actb mRNA. Data represent 5 independent experiments with each group assayed in triplicate. ${ }^{\star} P<0.01$ versus HVJ-E only. (C) Immunoblot analysis of LPCAT1 from whole cell lysates described in B with $\alpha$-LPCAT1 antibody at days 3 and 7 after siRNA administration. (D) Primary alveolar type II cells were cultured in the presence of siRNAs as outlined in $\mathbf{B}$ and labeled with $\left[{ }^{3} \mathrm{H}\right] \mathrm{palmitic}$ acid $\left({ }^{3} \mathrm{H}-\mathrm{PA}\right)$. Palmitic acid incorporation into SatPC was measured and normalized to genomic DNA. Depletion of $L p c a t 1 \mathrm{mRNA}$ significantly reduced $\left[{ }^{3} \mathrm{H}\right]$ palmitate incorporation into SatPC. Data represent 3 independent experiments; ${ }^{*} P<0.01$ versus control siRNA or HVJ-E only. (E) MLE15 cells expressing empty vector or LPCAT-HA were labeled with [ $\left.{ }^{3} \mathrm{H}\right]$ palmitic acid. Palmitic acid incorporation into SatPC was significantly increased by expression of LPCAT1. Right: Increased expression of LPCAT1 in transfected cells. Data are pooled from 3 independent experiments, each performed in triplicate; ${ }^{*} P=0.006$ versus EV.

demonstrate that LPCAT1 is a single-pass type II transmembrane protein residing in the ER and that the catalytic domain, including the critical residue His135, is located in the ER lumen (Figure 2D).

$L P C A T 1$ is required for SatPC synthesis in vitro. We have previously shown that Lpcat1 mRNA is localized to the distal epithelium of embryonic and adult mouse lung (18). To assess the pattern of LPCAT1 protein expression in mouse lung, we generated a polyclonal antibody against recombinant mouse LPCAT1 C terminus (amino acids 413-534). Immunohistochemistry of adult mouse lung demonstrated that LPCAT1 protein is restricted to alveolar type II cells (Figure 3A).

In order to define the function of LPCAT1 in vitro, LPCAT1 expression was suppressed in mouse primary alveolar type II cells by siRNA delivered via hemagglutinating virus of Japan envelopes (HVJ-E). Importantly, the type II cells were cultured on 100\% Matrigel to ensure maintenance of the differentiated phenotype (25). A $70 \%$ reduction in Lpcat 1 mRNA was observed 72 hours after transfection with Lpcat1-directed siRNA compared with a scrambled control siRNA or HVJ-E only, resulting in a significant decrease in LPCAT1 protein (Figure 3, B and C). LPCAT1 protein levels recovered to control levels by day 7 in culture (Figure 3C). Levels of $S f t p c$ mRNA, another type II cell-specific protein, were unaffected by Lpcat1 siRNA, as were the mRNA levels of Fasn and Scd1 (Figure 3B) and Srebf1 and Cebpa (data not shown), all of which are important for lipogenesis in alveolar type II cells (26). To determine whether LPCAT 1 is required for SatPC synthesis, primary type II cells were cultured in the presence of Lpcat1 siRNA for 72 hours, followed by labeling for an additional 14 hours with $\left[{ }^{3} \mathrm{H}\right]$ palmitic acid, the preferred acyl donor for LPCAT1 (18). The cells and media were harvested, then the number of radioactive counts incorporated into SatPC was determined. Palmitic acid incorporation into SatPC was decreased 2.7-fold in LPCAT1-suppressed cells compared with cells treated with scrambled control siRNA (con siRNA) or HVJ-E alone (Figure 3D). Similar decreases were observed in SatPC isolated from the medium of Lpcat1 siRNA-suppressed cells (data not shown). To determine whether increased expression of LPCAT1 is sufficient to increase SatPC synthesis in vitro, we transfected MLE15 cells with LPCAT-HA and performed the $\left[{ }^{3} \mathrm{H}\right]$ palmitic acid incorporation experiment as described for primary type II cells. Overexpression of LPCAT1 resulted in a 1.8-fold increase in palmitic acid incorporation into SatPC in cells (Figure 3E) and medium (data not shown), demonstrating that an increase in LPCAT1 is sufficient to increase SatPC synthesis.

Characterization of Lpcat ${ }^{G T /+} E S$ cells and generation of mice. To determine the function of LPCAT1 in vivo, we obtained an ES cell line that expressed a mutant allele of Lpcat1. These ES cells harbored a genetrap (GT) cassette in intron 9 of the Lpcat1 locus (Figure 4A). Processing of the mRNA transcribed from this locus resulted in 
A
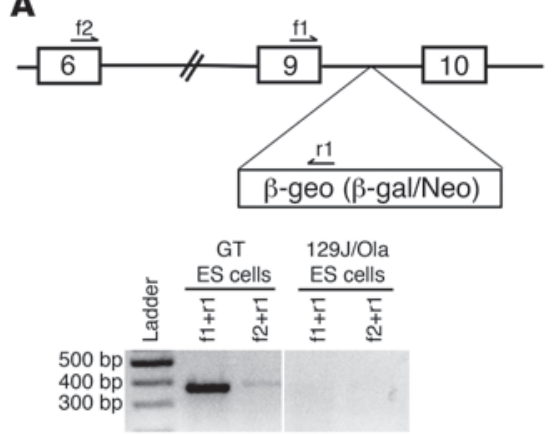

B

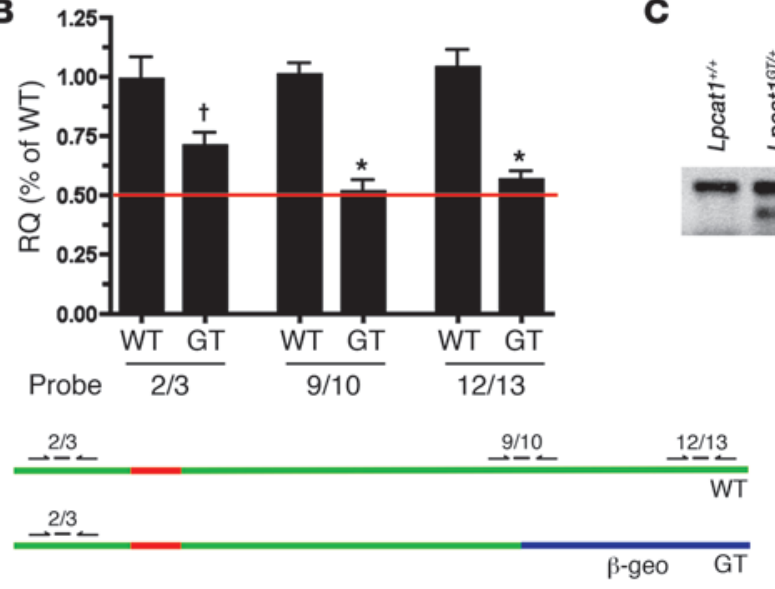

Figure 4

Confirmation of Lpcat1 GT in ES cells and generation of mice. (A) Top: Diagram of Lpcat1 locus. Boxes denote exons, and intervening lines denote introns. The $\beta$-geo selection cassette encoding a $\beta$-galactosidase/neomycin fusion protein was inserted in intron 9 , approximately 400 bp 3' of exon 9. f1, f2, and r2 denote primers used to confirm the presence of GT cassette in the Lpcat1 locus by qPCR analysis. Bottom: PCR analysis of the Lpcat1 locus in GT ES cells or parental ES cells (129J/Ola) using 2 separate primer sets. The presence of amplicons in GT ES cells with the absence of signal in parental cells demonstrates trapping of the Lpcat1 locus at exon 9. Vertical line indicates discontinuous lanes in the same gel. (B) qPCR analysis of GT ES cells. cDNA from GT ES cells (GT) and parental cells (WT) was subjected to qPCR analysis using 3 separate exon-spanning TaqMan primer/probe sets for Lpcat1 as depicted in the bottom panel: 2/3, 9/10, and 12/13. Data were normalized to Actb and represent 3 independent experiments, each performed in triplicate. Note the difference in signal between $2 / 3$ probe and $9 / 10$ probe in GT cells. ${ }^{\dagger} P<0.05$ versus $9 / 10$ and $12 / 13$ GT; ${ }^{*} P<0.05$ versus $2 / 3$ WT. RQ, relative quantification. (C) Multiplex PCR genotyping of genomic DNA from progeny of $L p c a t 1 G T /+$ intercrosses. Amplicon for WT allele is approximately $350 \mathrm{bp}$; GT, approximately $300 \mathrm{bp}$.

splicing of the $\beta$-galactosidase/neomycin cassette to exon 9 of Lpcat1, generating a LPCAT $1 / \beta$-geo fusion protein (Figure $4 \mathrm{~B}$ ). Insertion of the GT cassette in intron 9 of the Lpcat 1 locus was confirmed by RT-PCR analysis of mRNA from GT ES cells (Figure 4A). These data were further confirmed using quantitative PCR analysis with 3 exon-spanning TaqMan primer/probe sets: $2 / 3$ probe (detects WT and GT mRNA), 9/10 probe (WT-specific), and 12/13 probe (WTspecific). As expected, Lpcat 1 levels were decreased by $50 \%$ in the GT ES cells compared with the WT cells as measured with the $9 / 10$ and $12 / 13$ probes (Figure 4B). Fusion of the $\beta$-geo cassette to the first 9 exons of Lpcat 1 mRNA resulted in destabilization of the GT mRNA by approximately $50 \%$ as determined by the difference in signal intensity from the $2 / 3$ probe in the GT versus WT cells (Figure 4B).

Chimeric male mice were generated from the GT cells and backcrossed to Black Swiss females to generate $L p c a t 1^{G T /+}$ mice. These mice were healthy and fertile, with no overt phenotype. Lpcat1 ${ }^{G T /+}$ mice were intercrossed, and analysis of 104 mice at E18.5 indicated that $L p c a t 1^{G T / G T}$ mice were obtained at expected Mendelian ratios, demonstrating that homozygosity of the GT allele does not result in embryonic lethality (data not shown). The precise insertion point of the GT cassette was identified by PCR, allowing for multiplex PCR genotyping (Figure 4C).

SatPC is decreased in lung tissue of E18.5 Lpcat $1^{\mathrm{GT} / \mathrm{GT}}$ mice. To assess the role of LPCAT1 in SatPC synthesis in vivo, we measured SatPC levels from lung tissue of $L p c a t 1^{G T / G T}$ mice at E18.5. SatPC levels were decreased approximately $30 \%$ in $L p c a t 1^{G T / G T}$ mice as compared with littermate controls (Figure 5A). No significant differences in the percent composition of phosphatidylglycerol, phosphatidylinositol, phosphatidylethanolamine, lysobisphosphatidic acid, and sphingomyelin were detected (data not shown).

Immunoblot analysis performed on lung homogenate from E18.5 Lpcat $^{G T / G T}$, Lpcat $^{G T /+}$, and $L p c a t 1^{+/+}$mice demonstrated that the fusion protein produced from the GT allele is undetectable with our LPCAT1 antibody (Figure 5C). This was as expected, since the portion of the protein used for production of this antibody is not included in the translated fusion protein. Levels of $S f t p b, S f t p c$, and $A b c a 3$, all which play critical roles in surfactant homeostasis, were analyzed in E18.5 Lpcat1 ${ }^{G T / G T}$ mice. No differences were noted for any of the 3 proteins at the mRNA level (Figure 5B), protein expression level (Figure 5C), or protein localization (Figure 5D). Lung morphology was not altered in $L p c a t 1^{G T / G T}$ mice. The absence of LPCAT1 immunoreactivity on lung sections of $L p c a t 1^{G T / G T}$ mice confirmed both the specificity of the antibody for full-length LPCAT1 protein and the absence of splicing around the GT cassette in $L p c a t 1^{G T / G T}$ mice (Figure 5D). Taken together, these data demonstrate that LPCAT1 plays a critical role in regulating SatPC synthesis in vivo and that lung structure, other surfactant phospholipids, and expression of proteins critical for surfactant homeostasis are unaltered at E18.5 despite a 30\% decrease in SatPC.

Lpcat $1^{G T / G T}$ mice show varying perinatal mortality. Of the 520 newborn pups generated from $L p c a t 1^{G T /+}$ intercrosses, approximately $27 \%$ of $L p c a t 1^{G T / G T}$ mice were either found dead or exhibited severe respiratory distress, including chest retractions and cyanosis, and succumbed within 4-6 hours postpartum (Figure 6A and Table 1). To determine the stability of the Lpcat1/B-geo mRNA in vivo, we performed quantitative real-time RT-PCR (qPCR) analysis on RNA isolated from lung tissue of $L p c a t 1^{G T / G T}$ mice. The absence of signal from the $12 / 13$ probe in $L p c a t 1^{G T / G T}$ mice confirmed the specificity of this probe for the WT allele and demonstrated that splicing around the GT cassette to produce a full-length mRNA did not occur (Figure 6B). In contrast, substantial variability in the signal generated with the $2 / 3$ probe was detected in $L p c a t 1^{G T / G T}$ mice, with lower Lpcat $1 \mathrm{mRNA}$ levels present in the dead or gasping mice (dead) compared with alive mice (i.e., no overt phenotype). 

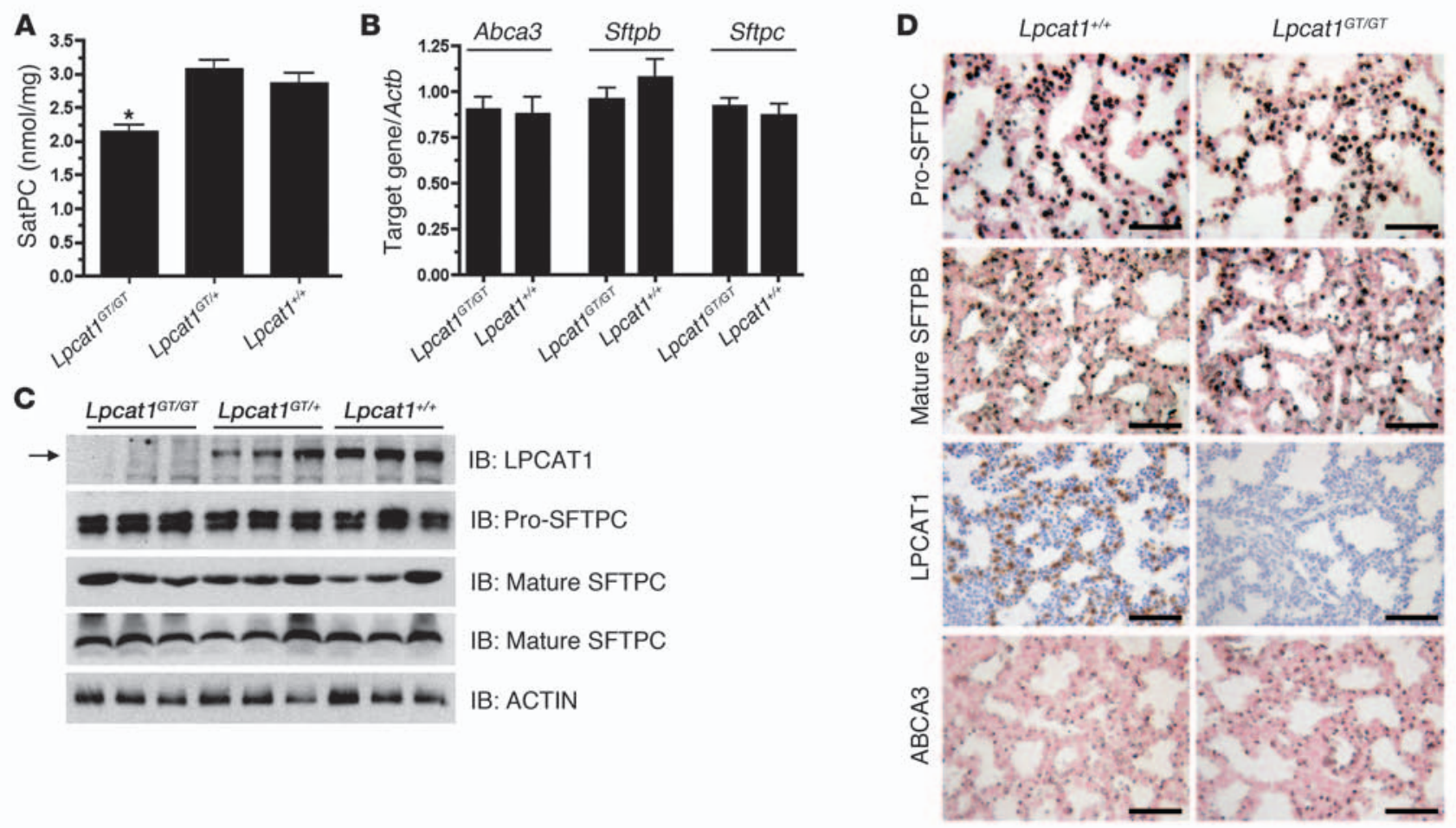

\section{Figure 5}

Lung SatPC content is decreased in E18.5 Lpcat1GT/GT mice. (A) Measurement of lung tissue SatPC content in E18.5 mice demonstrates that Lpcat1GT/GT lungs contain significantly less SatPC. Data represent $n=5$ per genotype and are normalized to tissue weight. ${ }^{*} P<0.01$ versus Lpcat1 ${ }^{+/+}$and Lpcat1 ${ }^{G T /+}$. (B) qPCR analysis of Abca3, Sttpb, and Sttpc from lung tissue of E18.5 mice shows that expression of these genes is unchanged. Data represent $n=5$ for each genotype and are normalized to Actb. (C) Immunoblot analysis of lung homogenate from $\mathrm{E} 18.5$ mice using antisera directed against the $C$ terminus of LPCAT1 $\left(\mathrm{M}_{\mathrm{r}} \sim 60 \mathrm{kDa}\right)$, pro-SFTPC $\left(\mathrm{M}_{\mathrm{r}} \sim 21 \mathrm{kDa}\right)$, the mature peptide of SFTPC (Mr $\left.\sim 4 \mathrm{kDa}\right)$, or the mature peptide of SFTPB $\left(\mathrm{M}_{\mathrm{r}} \sim 16 \mathrm{kDa}\right.$, nonreduced). The blot for mature SFTPB was stripped and reprobed with $\alpha$-ACTIN as a loading control. Note the lack of LPCAT1 immunoreactivity in $L p c a t 1 G T / G T$ mice (arrow) and no change in pro-SFTPC, mature SFTPC, or mature SFTPB protein. (D) Immunohistochemical analysis of pro-SFTPC, mature SFTPB, LPCAT1, and ABCA3 in lung sections from $L p c a t 1^{G T / G T}$ and $L p c a t 1^{+/+}$ mice confirms the results in C. Scale bars: $50 \mu \mathrm{m}$.

Lung tissue SatPC levels were significantly decreased in both Lpcat $1^{\text {GT/GT }}$ dead (46.0\% of WT) and Lpcat $1^{\text {GT/GT }}$ alive (60.0\% of WT) mice compared with $L p c a t 1^{G T /+}$ and $L p c a t 1^{+/+}$littermates (Figure $6 \mathrm{C})$. Notably, when the tissue SatPC data were plotted against Lpcat 1 mRNA levels obtained from the same animals, a strong correlation existed, suggesting that the incomplete penetrance of phenotype observed in newborn Lpcat $1^{G T / G T}$ mice is due to variability in stability of the Lpcat $1 / \beta$-geo mRNA and/or activity of the LPCAT $1 / \beta$-geo fusion protein (Figure $6 \mathrm{D}$ ). Acyltransferase activity assays were performed on lung microsomes from newborn Lpcat $1^{G T / G T}$ mice and littermate controls to determine whether the LPCAT $1 / \beta$-geo fusion protein was catalytically active. The data show that Lpcat1 ${ }^{G T / G T}$ mice retain significant acyltransferase activity (Figure 6E) and this activity strongly correlated with tissue SatPC levels, as was the case with Lpcat1 mRNA expression (Figure 6F). In mice, Lpcat1 mRNA expression is maximal at E18.5 (18). Consistent with these data, tissue SatPC levels increased 3.3-fold from E18.5 to P1 in Lpcat $1^{+/+}$mice (Figure 6G), while the fold increase in $L p c a t 1^{G T / G T}$ dead or alive mice was only 2.0 and 2.6, respectively. Collectively, these data demonstrate that LPCAT1 plays a pivotal role in SatPC production in the lung and that the level of Lpcat1 mRNA and acyltransferase activity correlate with tissue SatPC levels and perinatal survival.
Surfactant protein expression in Lpcat $1^{G T / G T}$ mice. Histopathology was performed on lung tissue of newborn $L p c a t 1^{G T / G T}$ mice that succumbed from RDS to determine the effects of decreased LPCAT1 on lung structure. While some areas of the lungs from $L p c a t 1^{G T / G T}$ mice that displayed respiratory distress were well inflated (Figure 7C, arrowhead) and comparable to those from asymptomatic Lpcat $1^{G T / G T}$ mice (Figure 7, B and E) and wild-type littermates (Figure 7, A and D), other areas showed collapsed airspaces and hemorrhagic regions and had proteinaceous material in the distal airways, consistent with respiratory distress (Figure 7C, arrow, and Figure 7F).

The levels of $S f t p b, S f p t c$, and Abca3 mRNA and ABCA3 protein were unchanged in $L p c a t 1^{G T / G T}$ RDS and alive mice compared with littermate controls (Figure 7J and Figure 7, G-I). However, both pro- and mature SFTPC proteins were decreased in the majority of Lpcat $^{{ }^{G T / G T}}$ animals (Figure $7 \mathrm{~K}$ ). While SFTPC is dispensable for the transition to air breathing in mice (27), SFTPB-deficient mice display profound neonatal respiratory distress and succumb shortly after birth (28). However, levels of mature SFTPB peptide were unchanged in the majority of Lpcat $1^{G T / G T}$ mice compared with littermate controls (Figure $7 \mathrm{~K}$ ). To determine whether lamellar body biogenesis was perturbed in Lpcat $1^{G T / G T}$ RDS mice, we performed ultrastructural analysis on lung tissue from newborn mice. The number, size, and morphology of lamellar bodies in alveolar type II 


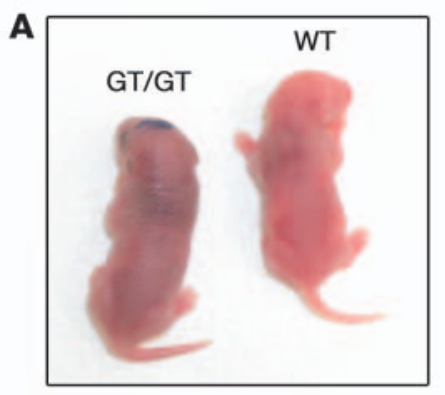

B
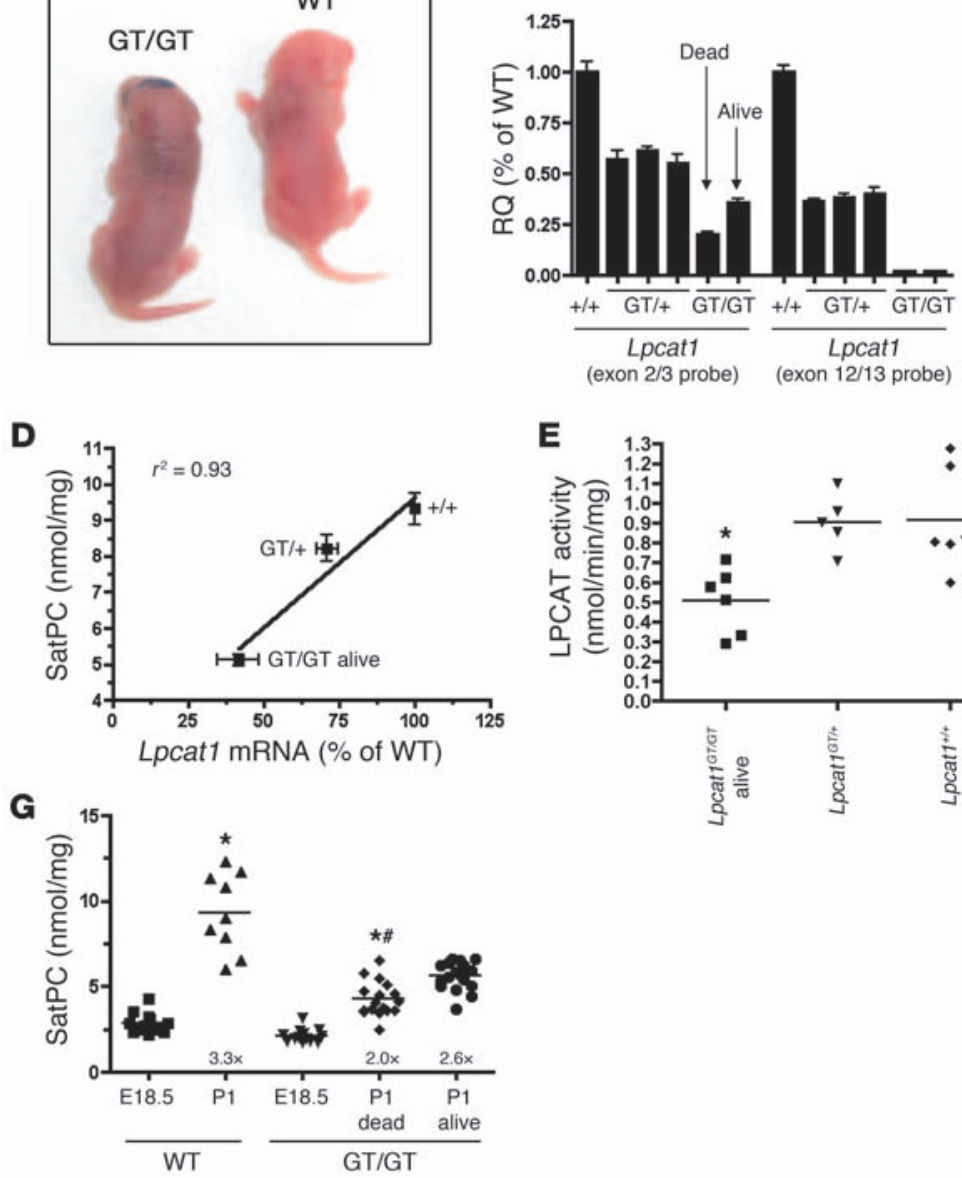

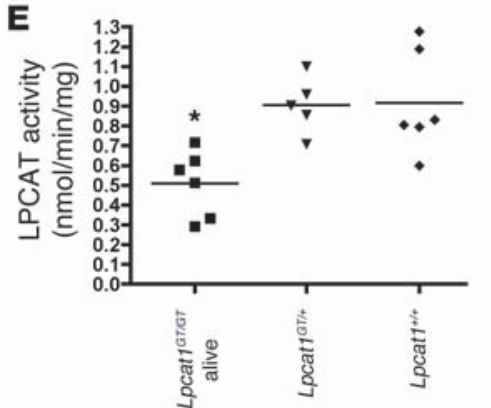

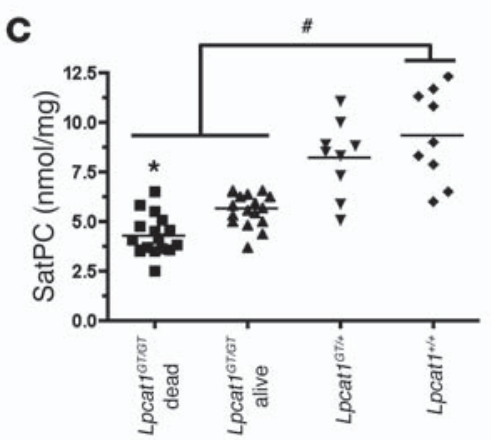

$\mathbf{F}$

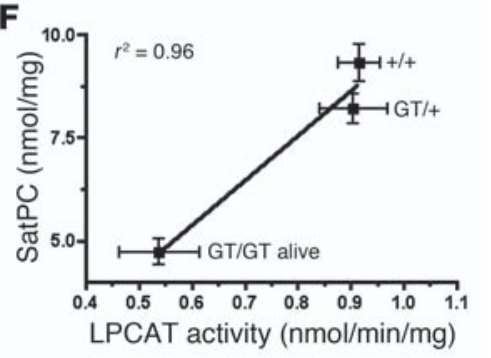

Figure 6

Perinatal mortality in Lpcat1GT/GT mice correlates with SatPC content and LPCAT1 activity. (A) Cyanotic appearance of a newborn Lpcat1GT/GT mouse compared with wild-type littermate. (B) qPCR analysis of lung tissue from individual newborn mice using 2 primer/probe sets for $L p c a t 1$. Data were normalized to Actb; each bar represents a single animal, and error bars represent error within technical replicates. Note the decreased amount of $L p$ cat 1 in dead versus alive mice as detected with an exon $2 / 3$ probe, suggesting variable mRNA stability. (C) Tissue SatPC content in newborn mice. Data represent $n=17$ per genotype for $L p c a t 1^{G T / G T}$ dead and alive, $n=9$ for $L p c a t 1^{G T /+}$ and $L p c a t 1^{+/+}$. ${ }^{*} P<0.05$ versus $L p c a t 1^{G T / G T}$ alive; $\# P<0.001$ versus $L p c a t 1 G T / G T$ dead and alive. (D) SatPC correlates with $L p c a t 1$ mRNA in newborn mice. Lpcat1 mRNA was detected by qPCR using the exon2/3 primer/probe set and normalized to Actb. Data represent $n=9$ for each genotype, and both assays were performed on lung tissue from the same animal. (E) Acyltransferase activity assays from lung tissue of newborn mice. LPcat1GT/GT mice had significantly less acyltransferase activity than controls. Data represent $n=5$ per genotype. ${ }^{*} P<0.05$ versus $L p c a t 1^{+/+}$mice. (F) SatPC content correlates with LPCAT1 activity in newborn mice. Data represent $n=5$ for each genotype. (G) Tissue SatPC levels in E18.5 and P1 mice. Note the 3.3-fold increase in SatPC in $L$ pcat $1^{+/+}$mice from E18.5 to P1 versus 2.0-fold and 2.6-fold increases in $L p c a t 1^{G T / G T}$ dead and alive mice, respectively. ${ }^{\star} P<0.001$ versus $\mathrm{E} 18.5$ of respective genotype; ${ }^{\#} P<0.01$ versus $\mathrm{P} 1$ Lpcat ${ }^{G T / G T}$ alive mice.

cells was similar in Lpcat $1^{\text {GT/GT }}$ RDS mice and $L p c a t 1^{+/+}$controls (Figure 7, L and M, arrowheads). Furthermore, abundant surfactant was found in the alveolar lumina of Lpcat1 ${ }^{\text {GT/GT }}$ RDS mice, including the presence of tubular myelin structures, despite the $53 \%$ decrease in SatPC in these animals (Figure 7, N and O, arrows).

Surfactant from newborn Lpcat $1^{G T / G T}$ mice has poor surface activity. The surface tension-lowering properties of the surfactant isolated from newborn mice were evaluated using a captive bubble surfactometer. While surfactant from $L p c a t 1^{G T /+}$ and $L p c a t 1^{+/+}$mice efficiently reduced the minimum surface tension below $5 \mathrm{mN} / \mathrm{m}$, the prototypical values of a high-quality surfactant, surfactant isolated from newborn $L p c a t 1^{G T / G T}$ alive mice, displayed a 2.9-fold increase to approximately $13 \mathrm{nM} / \mathrm{m}$ over Lpcat $1^{\mathrm{GT} /+}$ and $L$ pcat $1^{+/+}$controls, indicative of poor surfactant function (Figure 8A).
To evaluate the level of phospholipid-associated mature SFTPB peptide in $L$ pcat $1^{G T / G T}$ mice, we performed immunoblot analysis on an aliquot of the same surfactant sample used for surface tension assays. As shown in Figure 8B, Lpcat $1^{G T / G T}$ mice had levels of phospholipid-associated mature SFTPB protein comparable to those of their littermate controls, consistent to what was observed in whole lung homogenate at E18.5 (Figure 5C) and P1 (Figure 7K). Notably, the surfactant from $L p c a t 1^{G T / G T}$ alive mice was functionally poor, even though SFTPB protein levels were normal (Figure 8, A and B). Together, these data indicate that the surfactant isolated from Lpcat $1^{G T / G T}$ mice is functionally poor, independent of phospholipidassociated SFTPB levels.

Characterization of the LPCAT1/ $\beta$-geo fusion protein. The LPCAT1/ $\beta$-geo cDNA was cloned from primary alveolar type II cells isolated 
Table 1

Mendelian distribution of newborn LpcatGT/GT, LpcatGT/t, and Lpcat $^{+/+}$mice

\begin{tabular}{lcccc} 
Genotype & Expected & $\begin{array}{c}\text { Collected } \\
\text { (total, 520) }\end{array}$ & $\begin{array}{c}\text { Dead } \\
(\boldsymbol{n})\end{array}$ & $\begin{array}{c}\text { Dead } \\
\text { (\% of genotype) }\end{array}$ \\
GT/GT & $25 \%$ & $119(23 \%)$ & 32 & 26.9 \\
GT/+ & $50 \%$ & $270(52 \%)$ & 6 & 2.2 \\
$+/+$ & $25 \%$ & $131(25 \%)$ & 4 & 3.1 \\
\hline
\end{tabular}

from adult $L p c a t 1^{G T /+}$ mice and placed in-frame with a C-terminal HA tag in a mammalian expression vector to characterize the function of the LPCAT1/ $\beta$-geo fusion protein. Results demonstrated that the LPCAT $1 / \beta$-geo protein localizes to the ER of transfected HeLa cells in a pattern identical to the WT protein (Supplemental Figure 1A; supplemental material available online with this article;
doi:10.1172/JCI38061DS1). Furthermore, the LPCAT1/ $\beta$-geo protein shows robust $\beta$-galactosidase activity in vitro, demonstrating that the LPCAT1/ $\beta$-geo transcript generates a functional protein (Supplemental Figure 1B). Due to a notable decrease in staining intensity observed in the immunofluorescence studies, levels of protein expression were quantitated by FACS analysis of transfected HeLa cells. LPCAT1/ $\beta$-geo-transfected cells showed decreased levels of expression compared with cells transfected with wild-type LPCAT1 or the H135A mutant, demonstrating increased turnover of this protein (Supplemental Figure 1, C and D). In acyltransferase assays, the LPCAT $1 / \beta$-geo fusion protein generated SatPC at a level intermediate to the wild-type protein and the H135A mutant (Supplemental Figure 1E) when normalized to protein expression levels (Supplemental Figure 1D). Finally, expression of the LPCAT $1 / \beta$-geo fusion protein in HeLa cells did not result in toxicity (Supplemental Figure 1F), and there was no evidence of an ER stress response in the lung tissue of $L p c a t 1^{G T / G T}$ mice at E18.5 as assessed by levels of Hspa5 (BiP) mRNA (Supplemental Figure
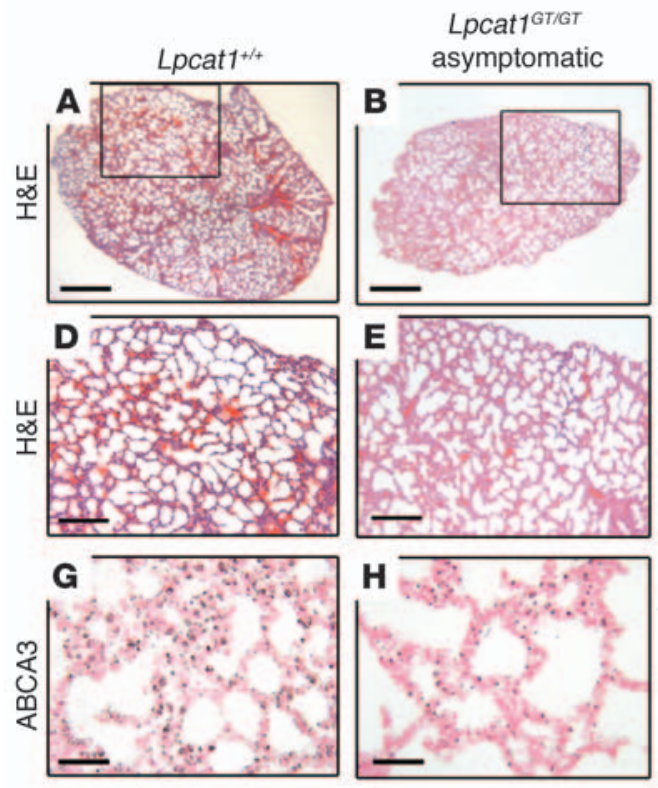

K

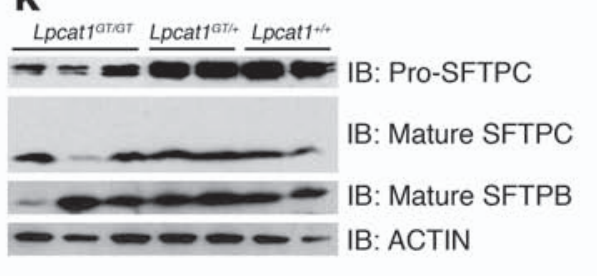

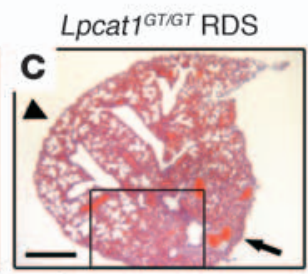
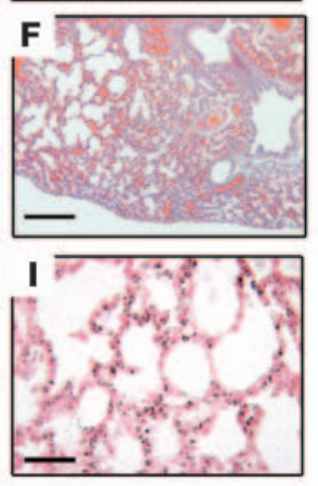
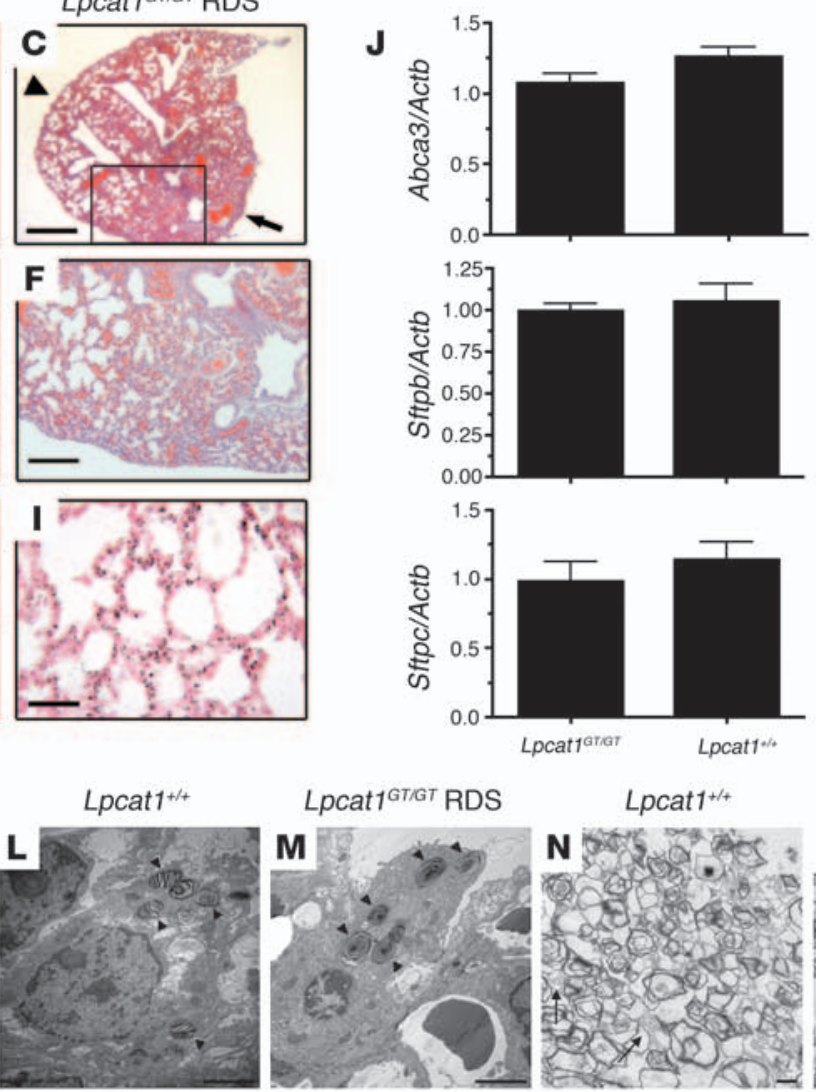
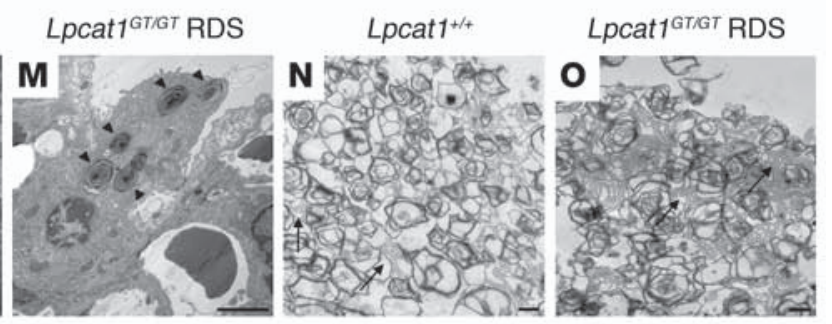

\section{Figure 7}

Analysis of surfactant-associated proteins in newborn Lpcat1 ${ }^{G T / G T}$ mice. (A-F). H\&E-stained lung tissue sections from newborn $L p c a t 1$ GT/GT mice that succumbed from RDS, showing areas of atelectasis and hemorrhaging (C, arrow) compared with less-affected areas in the same lung (C, arrowhead) and lungs of asymptomatic $L p c a t 1^{G T / G T}(\mathbf{B})$ and wild-type littermates (A). (D-F) High-power magnifications of areas boxed in A-C. ABCA3 protein levels were assessed by immunostaining (G-I). Scale bars: $500 \mu \mathrm{m}$ (A-C), $100 \mu \mathrm{m}$ (D-F), $50 \mu \mathrm{m}$ (G-I). (J) qPCR analysis of $A b c a 3$, Sttpb, and Sftpc from lung tissue of newborn mice. Data represent $n=5$ for each genotype and were normalized to Actb. (K) Immunoblot analysis of lung homogenate from newborn mice using antisera directed against pro-SFTPC $\left(\mathrm{M}_{\mathrm{r}} \sim 21 \mathrm{kDa}\right)$, the mature peptide of SFTPC (M $\sim 4 \mathrm{kDa})$, or the mature peptide of SFTPB $\left(\mathrm{M}_{\mathrm{r}} \sim 16 \mathrm{kDa}\right.$, nonreduced). The blot for mature SFTPB was stripped and reprobed with $\alpha-A C T I N$ as a loading control. (L-O) Representative transmission electron micrograph of lung tissue from a newborn Lpcat1GT/GT RDS mouse and a Lpcat1 ${ }^{+/+}$ littermate control. Note the numerous lamellar bodies in alveolar type II cells (arrowheads, $\mathbf{L}$ and $\mathbf{M}$ ) and abundant luminal surfactant and tubular myelin structures (arrows, $\mathbf{N}$ and $\mathbf{O}$ ) in both genotypes. Scale bars: $2 \mu \mathrm{m}(\mathbf{L}-\mathbf{O})$. 


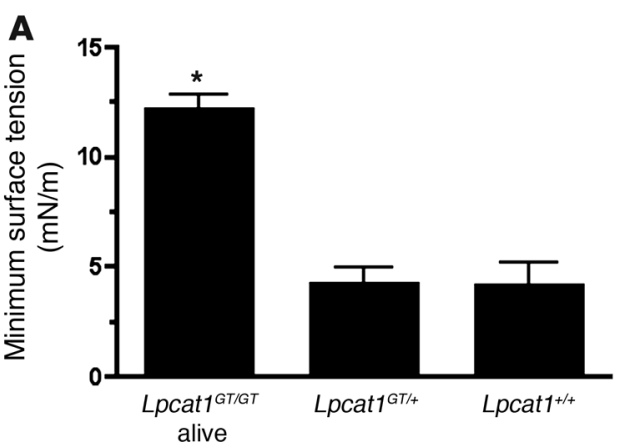

B
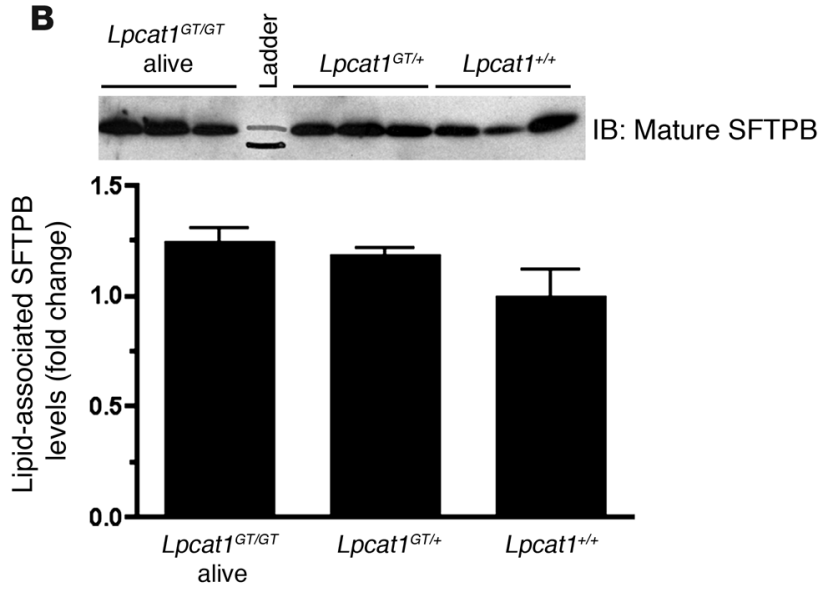

$1 \mathrm{G})$. Collectively, these data demonstrate that the LPCAT $1 / \beta$-geo mutation generates an ER-localized fusion protein that retains significant acyltransferase activity in vitro but is less stable than the wild-type protein. Finally, the LPCAT1/ $\beta$-geo protein does not result in overt cell toxicity when expressed at high levels in vitro, nor does it elicit an ER stress response in vivo.

\section{Discussion}

SatPC is the phospholipid species most responsible for the surface tension-lowering properties of pulmonary surfactant, thereby preventing alveolar collapse at end-expiration. Alveolar SatPC is synthesized by 2 pathways: de novo synthesis or a remodeling mechanism involving phospholipase-mediated deacylation of monosaturated PC, followed by reacylation with palmitoyl-CoA by a lysoPC acyltransferase. The 2 other less-abundant species of surfactant-associated PC, namely 16:0/14:0 and 16:0/16:1 PC, likely also arise via this remodeling pathway associated with LPCAT1 (ref. 19 and our unpublished observations). It is well established that lysoPC acyltransferase activity exists in the lung (29-34), and 3 lines of evidence from previous studies suggested that LPCAT1 may be the lysoPC acyltransferase involved in the biogenesis of alveolar SatPC. First, Lpcat 1 mRNA is developmentally regulated in the mouse lung, with peak expression at E18.5, and is restricted to alveolar type II cells (18). Second, Lpcat 1 mRNA is induced by KGF and dexamethasone in vitro (18), both of which stimulate phospholipid synthesis in type II cells $(22,35)$. Third, LPCAT1 preferentially acylates lysoPC with palmitate (16:0) or myristate (14:0) in transiently transfected cells $(18,19)$. However, LPCAT1 can utilize 18:2 and 18:1 as acyl donors when expressed at supraphysiological levels (19), indicating that this enzyme may also contribute PC

\section{Figure 8}

Surfactant isolated from $L p c a t 1^{G T / G T}$ mice fails to reduce minimum surface tension to wild-type levels. (A) Surface tension-lowering properties of lung surfactant isolated from newborn mice was measured on a captive bubble surfactometer. Minimum surface tensions of surfactant preparations isolated from $L p c a t 1^{G T / G T}$ alive mice were significantly higher than those of $L p c a t 1^{G T /+}$ and $L p c a t 1^{+/+}$mice. Data represent at least $n=5$ per genotype. ${ }^{*} P<0.001$ versus $L p c a t 1^{+/+}$and $L p c a t 1^{G T /+}$. (B) Immunoblot analysis of phospholipid-associated mature SFTPB from an aliquot of samples used for captive bubble surfactometer analysis in A. Input was normalized to $40 \mathrm{nmol}$ of total phospholipid. Graph represents densitometry of immunoblot. Levels of surfactant-associated, mature SFTPB protein were similar among all genotypes.

to cellular membranes. The results from the present study confirm and extend previous in vitro data by demonstrating that (a) LPCAT1 is an ER-resident type II transmembrane protein with a catalytic motif containing His135 in the ER lumen; (b) LPCAT1 regulates SatPC synthesis in primary cultures of alveolar type II cells; and (c) Lpcat1 mRNA content and acyltransferase activity correlate directly with SatPC content and survival in Lpcat $1^{G T / G T}$ mice. Collectively, these data demonstrate that LPCAT1 is required for the transition to air breathing and underscore the importance of the remodeling pathway to SatPC synthesis in vivo.

One advantage of the hypomorphic Lpcat1/ $\beta$-geo allele was that it allowed us to define the critical amount of SatPC required for the transition to air breathing. Specifically, the differences in SatPC levels in the newborn $L p c a t 1^{G T / G T}$ dead versus alive animals were small (46.0\% of wild-type versus $60.0 \%$ of wild-type, respectively; Figure $6 \mathrm{C})$, suggesting that the threshold level of SatPC required for perinatal lung function lies somewhere between 4.3 and $5.6 \mathrm{nmol} / \mathrm{mg}$ tissue weight. These data are consistent with lung function studies performed in preterm rabbits, where lung compliance failed to improve with increasing amounts of alveolar SatPC until a threshold value of $0.7 \mu \mathrm{mol} / \mathrm{kg}$ body weight was reached (36). Beyond this threshold, compliance rapidly reached maximum values with minimal increases in SatPC pool size, demonstrating a nonlinear relationship between SatPC content and lung compliance.

The importance of SatPC in the transition to air breathing is supported by data from several species (including rat, rabbit, lamb, monkey, and human) demonstrating that SatPC levels increase markedly at term (37). Our data presented in this study are consistent with these findings, as we observed a 3.3-fold increase in SatPC from E18.5 to P1 in wild-type mice (Figure 6G). In contrast, the increase in SatPC during this transition was markedly lower in $L p c a t 1^{G T / G T}$ mice (2.0- to 2.6 -fold in dead vs. alive mice, respectively). Furthermore, $L p c a t 1^{G T / G T}$ mice that survived the transition to air breathing were asymptomatic out to 7 weeks of age, despite a $40 \%$ reduction in tissue SatPC levels at this age (data not shown). Together, these data suggest that full LPCAT1 activity may only be necessary to generate the high SatPC levels required for the transition to air breathing and that decreased LPCAT1 activity may permit adequate adult lung function under normal conditions. This hypothesis remains to be formally tested.

It is important to note that the lungs of dead Lpcat $1^{G T / G T}$ mice retained substantial acyltransferase activity compared with control littermates (Figure 6E). Since the LPCAT1/ $\beta$-geo fusion protein generated from the GT allele contains the catalytic motif encoded by exon 3 , including the critical residue His 135 , one possible explanation is that the LPCAT $1 / \beta$-geo fusion protein retains 
acyltransferase activity. To test this hypothesis, the LPCAT $1 / \beta$ geo cDNA was cloned from $L p c a t^{G T /+}$ type II cells and functionally characterized in vitro. The data (Supplemental Figure 1) demonstrate that the LPCAT $1 / \beta$-geo protein is considerably less stable that the wild-type protein, in concordance with what was observed in vivo at the mRNA level (Figure 6B). Nonetheless, the LPCAT $1 / \beta$-geo protein localized to the ER and retained considerable acyltransferase activity when normalized to protein expression. Importantly, the LPCAT $1 / \beta$-geo fusion protein was not toxic to transfected HeLa cells and did not elicit and ER stress response in vivo. These data demonstrate that the LPCAT $1 / \beta$-geo allele generates a hypomorphic protein that most likely accounts for the variability in phenotype observed in vivo. Our data in isolated type II cells demonstrating that palmitic acid incorporation into SatPC was decreased $63 \%$, with a $70 \%$ suppression of Lpcat1 mRNA (Figure 3) support the idea that LPCAT1 was incompletely activated in $L p c a t 1^{G T / G T}$ mice and suggest that complete inactivation of the Lpcat1 allele will result in uniform mortality. This possibility is currently under investigation.

An alternative explanation for the remaining activity in $L p c a t 1^{G T / G T}$ mice is the existence of additional lysoPC acyltransferase(s) that generate SatPC. Recently, 3 additional lysoPC acyltransferases, LPCAT2, LPCAT3, and LPCAT4, have been identified and partially characterized (38-43). LPCAT2 also generates SatPC from lysoPC and palmitoyl-CoA and is $48 \%$ homologous to LPCAT1. Lpcat2 mRNA is present in the lung, although its expression appears to be restricted to alveolar macrophages (40). Furthermore, transcripts for LPCAT 2 in lung tissue were only $1.5 \%$ as abundant as LPCAT1 and were not elevated in Lpcat ${ }^{G T / G T}$ mice (data not shown), making it unlikely that LPCAT2 functionally compensates for decreased LPCAT1 activity. LPCAT3 is also expressed in human adult lung. It has been shown to acetylate LysoPC with palmitoylCoA when overexpressed in yeast that is LPCAT deficient (42) but not in mammalian CHO cells (39). While LPCAT4 is expressed at low levels in the human and mouse lung (43), this enzyme does not acylate LysoPC with palmitoyl-CoA in vitro (39). The possible contribution of LPCAT3 and LPCAT4 to SatPC generation in vivo remains to be determined.

PC is the most abundant phospholipid of mammalian cells and is particularly enriched in ER and mitochondrial membranes as well as the plasma membrane (44). Choline cytidylyltransferase $\alpha$ (CCT $\alpha$, gene symbol Pcyt1a), the primary rate-limiting enzyme in the de novo synthesis of all PC species, is absolutely required for embryogenesis, since global deletion of Pcyt1a in the mouse results in embryonic lethality prior to implantation (45). Interestingly, adult $P c y t 1 a^{+/-}$mice displayed a slight reduction in total PC content in the lung, but not in the liver, suggesting a role for CCT $\alpha$ in surfactant phospholipid synthesis. Consistent with this concept, deletion of Pcyt1a in pulmonary epithelial cells resulted in lethal neonatal respiratory distress in mice associated with a $40 \%$ decrease in total lung PC (46). However, this study did not address the direct impact of decreased SatPC versus other PC species on the lethal respiratory phenotype.

While some of the SatPC in alveolar type II cells is synthesized de novo (12), the majority is generated via the remodeling pathway, with estimates ranging between $55 \%$ and $75 \%(12,13)$. The first enzymatic reaction in the remodeling pathway is the deacylation of PC by $\mathrm{PLA}_{2}$. Numerous $\mathrm{PLA}_{2}$ isoforms exist in the mammalian genome $(47,48)$, and $\mathrm{PLA}_{2}$ activity has been localized to distinct subcellular compartments of the lung, including cytosol, microsomes, lysosomes, and lamellar bodies (49-56). PRDX6 is a Ca ${ }^{2+}$-independent, acidic $\mathrm{PLA}_{2}$ that localizes to lysosomes and lamellar bodies and is a dual-function enzyme with peroxidase and phospholipase activity (57). $\operatorname{Prdx6^{-/-}}$ mice are viable and fertile (58) and accumulate total phospholipids, PC, and SatPC in lung tissue and lavage as a function of age, demonstrating a role for this enzyme in surfactant phospholipid catabolism (59). However, the fact that $P r d x 6^{-/-}$mice do not phenocopy Lpcat ${ }^{G T / G T}$ mice suggests that other $\mathrm{PLA}_{2}$ enzymes may be operating in the deacylation arm of the remodeling pathway to generate SatPC.

Surfactant isolated from asymptomatic Lpcat $1^{G T / G T}$ newborn mice failed to reduce surface tension below $5 \mathrm{mN} / \mathrm{m}$, despite normal levels of SFTPB protein (Figure 8, A and B). Because SFTPB levels were normal in $L p c a t 1^{G T / G T}$ mice at E18.5 and P1, we believe that the marked elevation in surface tension in these animals $(\sim 13$ $\mathrm{mN} / \mathrm{m})$ is primarily due to decreased SatPC content in the surfactant. For comparison, the minimum surface tension values of surfactant isolated from adult mice conditionally deficient in SFTPB range from 12 to $18 \mathrm{mN} / \mathrm{m}$ (60), which is higher than what we observed in $L p c a t 1^{G T / G T}$ mice. It is important to note that the high surface tension measurements were detected in SFTPB deficient animals prior to visible signs of respiratory distress, demonstrating that values above $13 \mathrm{mN} / \mathrm{m}$ are compatible with life. Although changes in surfactant lipid components other than SatPC are known to alter surface tension properties, we detected no significant differences in the percent composition of phosphatidylglycerol, phosphatidylinositol, phosphatidylethanolamine, lysobisphosphatidic acid, and sphingomyelin in Lpcat $1^{G T / G T}$ mice (data not shown). While adding exogenous SatPC to the surfactant from newborn Lpcat $1^{G T / G T}$ mice should, in theory, reduce the minimal surface tension to WT levels, we predict from our previous experience that the addition of exogenous SatPC in an organic suspension to the pelleted endogenous surfactant suspension from Lpcat $1^{G T / G T}$ mice would not fully induce the required surfactant lipid-protein associations to restore minimum surface tension, therefore confounding interpretation of these results.

While we cannot definitively explain why $L p c a t 1^{G T / G T}$ mice survive the perinatal period despite high surface tension values, these data demonstrate that a primary defect in SatPC synthesis, with no changes in SFTPB content, results in a poor surfactant and suggests that the minimum surface tension value required for the transition to air breathing in mice is at least $13 \mathrm{mN} / \mathrm{m}$. Data from premature rabbit models have clearly shown that the relationship between SatPC content and lung compliance is not linear (36). Increasing SatPC content has little effect on compliance until a threshold is reached, after which maximal compliance is achieved and there is no additional benefit of further increases. We believe that the SatPC levels in hypomorphic Lpcat $1^{G T / G T}$ pups range just above (live) or below (die) this threshold. These data demonstrate that the primary cause of the lethal respiratory distress phenotype in the Lpcat $1^{G T / G T}$ mice that died was insufficient SatPC levels required for the transition to air breathing. To our knowledge, this is the first report of lethal neonatal respiratory distress with a primary defect in pulmonary SatPC synthesis.

Mutations in genes essential for the production and function of pulmonary surfactant, including $S f t p b, S f t p c$, and $A b c a 3$, have been linked to acute and chronic lung disease in children and adults (6). Given the critical requirement for LPCAT1 in the transition to air breathing, it is tempting to speculate that decreased LPCAT1 expression, resulting from mutations in either the regulatory 
or coding region of Lpcat1, may be responsible for the fatal RDS observed in a subset of newborn infants who have normal levels of SFTPB, SFTPC, and ABCA3. A total of 7 SNPs in the human Lpcat1 locus have been deposited in the NCBI Single Nucleotide Polymorphism database (www.ncbi.nlm.nih.gov/snp/, Build 130). Three of the 7 are synonymous mutations, 3 are missense mutations, and 1 results in a frameshift. While none of mutations have been associated with clinical disease, 4 of the 7 mutations cluster within $30 \mathrm{bp}$ of the critical $\mathrm{HX}_{4} \mathrm{D}$ motif in exon 3 , suggesting that these mutations may impact LPCAT1 function.

Surfactant deficiency also occurs in children and adults with ALI, and exogenous surfactant replacement therapies have not been conclusively efficacious in either population (61). Numerous factors have been proposed to explain the variability in efficacy, including etiology of injury, dosing regimen, surfactant composition, rapid clearance of exogenous surfactant in the mature lung, and delivery to injured areas of the lung (62-64). Overexpression of LPCAT1 in transiently transfected MLE15 cells was sufficient to increase the synthesis and secretion of SatPC (Figure 3E). Identifying the transcriptional networks that induce LPCAT1, including the candidates CEBP $\alpha$ and SREBF1, which are known to induce phospholipid synthesis in alveolar type II cells $(21,22,26,65)$, may allow for the increased production of endogenous SatPC in the injured lung, thereby providing an alternative therapeutic approach to the treatment of ALI.

In summary, we have shown that LPCAT1 is an ER-localized transmembrane protein that requires His 135 for its catalytic activity. LPCAT1 is critical for the production of SatPC in mouse alveolar type II cells and is required for the burst in SatPC synthesis needed for the transition to air breathing. These data highlight the importance of the remodeling pathway in SatPC synthesis in vivo and suggest that LPCAT1 may be an important biological target for therapeutic interventions in surfactant-associated deficiencies.

\section{Methods}

Expression constructs. Two epitope-tagged LPCAT1 constructs were generated by PCR amplification from mouse alveolar type II cell cDNA: LPCAT-HA, with a C-terminal HA tag, and LPCAT-FLAG, with an N-terminal FLAG tag. The amplicons were ligated into pcDNA3.1+ (Invitrogen). The H135A point mutant of mouse LPCAT1 was generated with the QuikChange kit (Promega) using LPCAT-HA/pcDNA3.1+ as a template.

Secondary structure prediction of mouse LPCAT1 protein. The primary amino acid sequence of mouse LPCAT1 protein was analyzed by the TMpred (http://www.ch.embnet.org/software/TMPRED_form.html), SOSUI (http://bp.nuap.nagoya-u.ac.jp/sosui/), and Kyte-Doolittle hydropathy plot (http://gcat.davidson.edu/rakarnik/kyte-doolittle.htm) algorithms.

Membrane fractionation assay. Experiments were performed using a method adapted from Schuberth and Buchberger (66). HEK293 cells (ATCC) were transfected with LPCAT-HA or LPCAT-FLAG using Lipofectamine 2000 (Invitrogen). The following day, cells were washed with PBS, scraped, and pelleted at $800 \mathrm{~g}$ for 10 minutes at $4^{\circ} \mathrm{C}$. The cell pellet was resuspended in $300 \mu \mathrm{l}$ of buffer A (50 mM Tris- $\mathrm{HCl}$ pH 7.5, $100 \mathrm{mM} \mathrm{NaCl}, 0.3 \mathrm{M}$ sorbitol, $1 \mathrm{mM}$ EDTA, and Complete protease inhibitors [Roche]), briefly sonicated, and split into 30- $\mu$ l aliquots. Membranes were pelleted from the aliquots by centrifugation at $20,000 \mathrm{~g}$ for 30 minutes at $4{ }^{\circ} \mathrm{C}$ and resuspended in buffer A only or buffer A containing one of the following additions: $0.6 \mathrm{M} \mathrm{NaCl}, 0.2 \mathrm{M} \mathrm{Na}_{2} \mathrm{CO}_{3}, 0.1 \%$ Triton X-100, or $1 \%$ Triton X-100. After 15 minutes of incubation on ice, the samples were centrifuged at $20,000 \mathrm{~g}$ for 30 minutes to separate pellet and soluble fractions, and partitioning of LPCAT1 was monitored by immunoblot analysis.
Trypsin protection assay. Membrane pellets were isolated as described for membrane fractionation experiments, except that buffer A lacked protease inhibitors. The membrane pellets were incubated with trypsin $(10 \mu \mathrm{g} / \mathrm{ml}$, New England Biolabs) or trypsin plus 1\% Triton X-100 for 15 minutes on ice. The reaction was terminated with the addition of $2 \mathrm{mM} \mathrm{PMSF}$, and the reaction products were analyzed by immunoblot analysis.

Generation of Lpcat $1^{G T /+}$ mice. ES cells harboring the GT cassette in intron 9 of the Lpcat1 locus (cell line ID: BC0405) were obtained from the Sanger Institute. The GT cassette consists of a splice acceptor site from the Engrailed gene fused to a neomycin resistance cDNA followed by $\beta$-galactosidase cDNA. For RT-PCR confirmation experiments, ES cells were cultured on gelatin-coated plates in the presence of leukocyte inhibitory factor (LIF), alongside parental ES cells derived from 129J/Ola mice. Chimeras were generated by blastocyst injections of the Lpcat $1^{G T /+}$ ES cells at the University of Cincinnati Gene Targeting Facility. Chimeric males were backcrossed to Black Swiss females to generate $L p c a t 1^{G T /+}$ mice, referred to as founder $\left(\mathrm{F}_{0}\right)$ mice. All of the studies detailed in this article were performed with $\mathrm{F}_{2}$ offspring from $\mathrm{F}_{1}$ intercrosses. Mice were maintained in cages containing high-efficiency particulate-filtered air in a barrier facility. Sentinel control mice housed alongside study mice were deemed pathogen-free, and all procedures were conducted under protocols approved by Cincinnati Children's Hospital Medical Center's Institutional Animal Care and Use Committee.

Acyltransferase activity assays. To measure acyltransferase activity of LPCAT1-transfected cells, the reaction mixture (200 $\mu$ l final volume) contained $80 \mathrm{mM}$ Tris- $\mathrm{HCl} \mathrm{pH} 7.5,5 \mathrm{mM} \mathrm{MgCl} 2,200 \mu \mathrm{M}$ lysoPC (Avanti Polar Lipids), $20 \mu \mathrm{M} 1-{ }^{14} \mathrm{C}$-palmitoyl-CoA (PerkinElmer Life Sciences), and $20 \mu \mathrm{g}$ of cell lysate. The reaction was initiated by the addition of the protein homogenate, allowed to proceed for 20 minutes at $25^{\circ} \mathrm{C}$, and terminated with the addition of chloroform/methanol (2:1, vol/vol). The lipids were extracted, and the amount of palmitate incorporation into SatPC was determined as previously described (18). To measure endogenous acyltransferase activity in lungs, microsomes were isolated from newborn mouse lungs using a previously described protocol (67), and acyltransferase assays were performed as described above, except an input of $100 \mu \mathrm{g}$ microsomal protein was used.

Generation of LPCAT1 antibody. The cDNA encoding amino acids 413-534 of mouse LPCAT1 protein was amplified from LPCAT1/pcDNA3.1+ and cloned into the pET41a+ vector (Novagen) to create LPCAT1 $1_{413-534 /}$ pET41a+. BL21(DE3) bacteria were transformed with LPCAT1 $1_{413-534} /$ pET41a+, grown to log phase, and then induced with $1 \mathrm{mM}$ IPTG for 4 hours to produce the recombinant protein. The protein was then isolated from lysed cells, purified on a nickel column, and injected into guinea pigs with adjuvant to generate polyclonal antibodies.

Immunoblot analysis. Lysate preparation from HEK293 cells, protein standardization, and immunoblot analysis were performed as previously described (68). Whole lung homogenate was prepared by briefly sonicating fresh lung tissue $(\sim 50 \mathrm{mg})$ in RIPA buffer containing Complete protease inhibitors (Roche). The homogenate was spun at $10,000 \mathrm{~g}$ for 5 minutes to clear debris, and the supernatant was used for protein standardization and immunoblot analysis. Lysates were separated on 12\% PAGEgels (Fisher Scientific) for LPCAT1 and 10\%-20\% gels for mature SFTPB, pro-SFTPC, mature SFTPC, and actin under reducing conditions (except for mature SFTPB). Polyclonal antisera for mature SFTPB, pro-SFTPC, mature SFTPC, ABCA3, and pan-ACTIN were purchased from Seven Hills Bioreagents.

Lung histology and immunohistochemistry. Tissues were fixed in $4 \%$ paraformaldehyde and embedded in paraffin, and 5 - $\mu \mathrm{m}$ sections were cut for H\&E staining or immunohistochemistry. Primary antibodies used were: mature SFTPB, pro-SFTPC, and ABCA3 (Seven Hills Bioreagents) and LPCAT1 (in-house). Biotinylated secondary antibodies were used with the $\mathrm{ABC}$ Vectastain kit (Vector Laboratories). DAB with nickel enhance- 
ment was used for mature SFTPB, pro-SFTPC, and ABCA3 staining, followed by a nuclear fast red counterstain; DAB was used for LPCAT1 with a hematoxylin counterstain.

Primary alveolar type II cell isolation, culture, and administration of siRNA. Mouse alveolar type II cells were isolated and cultured on 100\% Matrigel (BD Biosciences) as previously described (69). ON-TARGETplus SMARTpool siRNA for mouse Lpcat1 (catalog L-059984-01) or a non-target control siRNA (catalog D-001600-01) were purchased from Dharmacon. siRNAs were delivered into primary type II cells via HVJ-E particles (CosmoBio, cata$\log$ SK-GN-001-EX) per the manufacturer's guidelines. Cells for biochemical analyses were harvested off the Matrigel with dispase (Gibco, Invitrogen).

Palmitic acid incorporation into SatPC. Mouse alveolar type II cells were isolated and cultured with Lpcat1 siRNAs as described above for 72 hours, then labeled with $1 \mu \mathrm{Ci} / \mathrm{ml}\left[{ }^{3} \mathrm{H}\right]$ palmitic acid for an additional 14 hours in a humidified incubator. The palmitic acid was stabilized in solution with $5 \%$ human serum albumin prior to use. Media and cells were harvested after labeling, and the lipids were extracted by the method of Bligh and Dyer (70). SatPC was isolated from each fraction using the method of Mason et al. (71). The amount of palmitic acid incorporated into SatPC was determined by quantification of radioactivity by liquid scintillation counting and normalized to nanograms of genomic DNA isolated from the cells using a previously described method (72). For labeling of MLE15 cells overexpressing LPCAT1, cells were labeled as described for type II cells 48 hours after transfection. SatPC was isolated from both medium and cellular fractions, and the data were normalized to cellular protein content determined by microBCA (Pierce).

Surface tension measurements of surfactant from newborn mice. Due to the inability to recover bronchoalveolar lavage fluid from newborn mice, surfactant was isolated by mechanically dicing newborn lungs into approximately $1-\mathrm{mm}^{3}$ pieces, followed by slowly stirring in $15 \mathrm{mM} \mathrm{NaCl}$ on a magnetic stirrer for 20 minutes at $2^{\circ} \mathrm{C}$. The lung tissue was removed from the suspension by filtering through 2 layers of gauze. The suspension was then centrifuged at 4,000 $\mathrm{g}$ over a $0.8-\mathrm{M}$ sucrose cushion for 15 minutes at room temperature, and large-aggregate surfactant was collected from the interface, diluted in $15 \mathrm{mM} \mathrm{NaCl}$, and centrifuged again at 40,000 $\mathrm{g}$ for 15 minutes (73). The large-aggregate surfactant pellet then was resuspended in a small amount of saline, and aliquots were used for phosphorous assay (74). The large-aggregate surfactant fractions from 3 mice for each group were used for minimal surface tension measurements using the captive bubble surfactometer (75). Three-microliter suspensions of large-aggregate surfactant fractions containing $15 \mu \mathrm{mol}$ of phospholipid were applied by microsyringe on the surface of a $25-\mu \mathrm{l}$ bubble. Sixty seconds after surfactant application, the bubble was oscillated 5 times, and minimal surface tensions at a surface area of $35 \%$ were measured.

Quantitative real-time PCR analysis. Total RNA for $\mathrm{qPCR}$ was isolated and reverse transcribed into cDNA by standard methods. qPCR was performed using TaqMan primer/probe sets (Applied Biosystems) specific for Sftpc (assay ID: Mm00488144_m1), Sftpb (assay ID: Mm00455681_m1), Abca3 (assay ID: Mm00550501_m1), Lpcat1 exons 2/3 (assay ID: Mm00628177_ m1), Lpcat1 exons 9/10 (assay ID: Mm00461015_m1), Lpcat1 exons 12/13 (assay ID: Mm01340302_g1), Hspa5 (assay ID: Mm00517691), Fasn (assay ID: Mm01253300_g1), and Scd1 (assay ID: Mm00772290_m1) and Actb (part number: 4352933E) as an internal control. The PCR reactions and relative quantifications were performed using $25 \mathrm{ng}$ of $\mathrm{cDNA}$ per reaction on a 7300 Real-Time PCR system (Applied Biosystems).

Transmission electron microscopy. The upper-right lobes of newborn mouse lungs were fixed, processed, and analyzed as previously described (76).

Statistics. All data are presented as mean \pm SEM, with $P \leq 0.05$ considered significant. Multiple group comparisons were made by 1-way ANOVA with Tukey's post-hoc analysis. Two-way comparisons were performed by 2 -tailed, unpaired Student's $t$ test. All analyses were performed using GraphPad Prism software version 4.0.

\section{Acknowledgments}

The authors thank Xiaofei Shangguan, Ross Ridsdale, Shawn Grant, and Angelica Falcone for excellent technical assistance and/or advice. This work is supported by NIH awards HL-084376 to J.M. Shannon and HL-029891 to J.M. Shannon and R.J. Mason. J.P. Bridges is supported through a postdoctoral fellowship from the Parker B. Francis Foundation.

Received for publication November 14, 2008, and accepted in revised form February 10, 2010.

Address correspondence to: John M. Shannon, Division of Pulmonary Biology, Cincinnati Children's Hospital Medical Center, 3333 Burnet Avenue, ML7059, Cincinnati, Ohio 45229, USA. Phone: 513.636.2938; Fax: 513.636.7868; E-mail: John.Shannon@cchmc.org.
1. Gregory TJ, et al. Surfactant chemical composition and biophysical activity in acute respiratory distress syndrome. J Clin Invest. 1991;88(6):1976-1981.

2. Schmidt R, et al. Time-dependent changes in pulmonary surfactant function and composition in acute respiratory distress syndrome due to pneumonia or aspiration. Respir Res. 2007;8:55.

3. Hallman M, Spragg R, Harrell JH, Moser KM, Gluck L. Evidence of lung surfactant abnormality in respiratory failure. Study of bronchoalveolar lavage phospholipids, surface activity, phospholipase activity, and plasma myoinositol. J Clin Invest. 1982; 70(3):673-683.

4. Pison $U$, et al. Surfactant abnormalities in patients with respiratory failure after multiple trauma. $A m$ Rev Respir Dis. 1989;140(4):1033-1039.

5. Gunther A, et al. Surfactant alterations in severe pneumonia, acute respiratory distress syndrome, and cardiogenic lung edema. Am J Respir Crit Care Med. 1996; 153(1):176-184.

6. Hamvas A, Cole FS, Nogee LM. Genetic disorders of surfactant proteins. Neonatology. 2007; 91(4):311-317.

7. Hawgood S, Clements JA. Pulmonary surfactant and its apoproteins. J Clin Invest. 1990;86(1):1-6.

8. Veldhuizen R, Nag K, Orgeig S, Possmayer F. The role of lipids in pulmonary surfactant. Biochim Biophys Acta. 1998;1408(2-3):90-108.

9. Possmayer F. Physicochemical aspects of pulmonary surfactant. In: Polin RA, Fox WW, Abman HS, eds. Fetal and Neonatal Physiology. Philadelphia, Pennsylvania, USA: Saunders; 2004:1014-1034.

10. Notter RH. Lung surfactants. Basic science and clinical applications. New York, New York: Marcel Dekker; 2000.

11. Goerke J. Pulmonary surfactant: functions and molecular composition. Biochim Biophys Acta. 1998; 1408(2-3):79-89.

12. den Breejen JN, Batenburg JJ, van Golde LM. The species of acyl-CoA in subcellular fractions of type II cells isolated from adult rat lung and their incorporation into phosphatidic acid. Biochim Biophys Acta. 1989;1002(3):277-282.

13. Mason RJ, Nellenbogen J. Synthesis of saturated phosphatidylcholine and phosphatidylglycerol by freshly isolated rat alveolar type II cells. Biochim Biophys Acta. 1984;794(3):392-402.

14. Post M, Schuurmans EA, Batenburg JJ, Van Golde LM. Mechanisms involved in the synthesis of disaturated phosphatidylcholine by alveolar type II cells isolated from adult rat lung. Biochim Biophys Acta. 1983;750(1):68-77.
15. Vereyken JM, Montfoort A, van Golde LM. Some studies on the biosynthesis of the molecular species of phosphatidylcholine from rat lung and phosphatidylcholine and phosphatidylethanolamine from rat liver. Biochim Biophys Acta. 1972;260(1):70-81.

16. Moriya T, Kano H. In vivo studies on the de novo synthesis of molecular species of rat lung lecithins. Tohoku J Exp Med. 1974;112(3):241-256.

17. Akino T, Abe M, Arai T. Studies on the biosynthetic pathways of molecular species of lecithin by rat lung slices. Biochim Biophys Acta. 1971;248(2):274-281.

18. Chen X, Hyatt BA, Mucenski ML, Mason RJ, Shannon JM. Identification and characterization of a lysophosphatidylcholine acyltransferase in alveolar type II cells. Proc Natl Acad Sci U S A. 2006; 103(31):11724-11729.

19. Nakanishi $\mathrm{H}$, et al. Cloning and characterization of mouse lung-type acyl-CoA:lysophosphatidylcholine acyltransferase 1 (LPCAT1). Expression in alveolar type II cells and possible involvement in surfactant production. J Biol Chem. 2006;281(29):20140-20147.

20. Shannon JM, Pan T, Nielsen LD, Edeen KE, Mason RJ. Lung Fibroblasts Improve Differentiation of Rat Type II Cells in Primary Culture. Am J Respir Cell Mol Biol. 2001;24(3):235-244.

21. Chang Y, Wang J, Lu X, Thewke DP, Mason RJ. KGF 
induces lipogenic genes through a PI3K and JNK/ SREBP-1 pathway in $\mathrm{H} 292$ cells. J Lipid Res. 2005; 46(12):2624-2635.

22. Chang Y, Edeen K, Lu X, De Leon M, Mason RJ. Keratinocyte growth factor induces lipogenesis in alveolar type II cells through a sterol regulatory element binding protein-1c-dependent pathway. $A m J$ Respir Cell Mol Biol. 2006;35(2):268-274.

23. Heath RJ, Rock CO. A conserved histidine is essential for glycerolipid acyltransferase catalysis. J Bacteriol. 1998;180(6):1425-1430.

24. Harayama T, Shindou H, Ogasawara R, Suwabe A, Shimizu T. Identification of a novel noninflammatory biosynthetic pathway of platelet-activating factor. J Biol Chem. 2008;283(17):11097-11106.

25. Deterding RR, Shannon JM. Proliferation and differentiation of fetal rat pulmonary epithelium in the absence of mesenchyme. J Clin Invest. 1995; 95(6):2963-2972.

26. Zhang F, Pan T, Nielsen LD, Mason RJ. Lipogenesis in fetal rat lung: importance of C/EBPalpha, SREBP-1c, and stearoyl-CoA desaturase. Am J Respir Cell Mol Biol. 2004;30(2):174-183.

27. Glasser SW, et al. Altered stability of pulmonary surfactant in SP-C-deficient mice. Proc Natl Acad Sci U S A. 2001;98(11):6366-6371.

28. Clark JC, et al. Targeted disruption of the surfactant protein B gene disrupts surfactant homeostasis, causing respiratory failure in newborn mice. Proc Natl Acad Sci U S A. 1995;92(17):7794-7798.

29. de Vries AC, Batenburg JJ, van Golde LM. Lysophosphatidylcholine acyltransferase and lysophosphatidylcholine: lysophosphatidylcholine acyltransferase in alveolar type II cells from fetal rat lung. Biochim Biophys Acta. 1985;833(1):93-99.

30. Batenburg JJ, Longmore WJ, Klazinga W, van Golde LM. Lysolecithin acyltransferase and lysolecithin: lysolecithin acyltransferase in adult rat lung alveolar type II epithelial cells. Biochim Biophys Acta. 1979; 573(1):136-144

31. Crecelius CA, Longmore WJ. Acyltransferase activities in adult rat type II pneumocyte-derived subcellular fractions. Biochim Biophys Acta. 1984;795(2):238-246.

32. Finkelstein JN, Maniscalco WM, Shapiro DL. Properties of freshly isolated type II alveolar epithelial cells. Biochim Biophys Acta. 1983;762(3):398-404.

33. Hoehn SK, Lesperance E, Klass DJ. Enzymes of phospholipid biosynthesis in rat alveolar type II cells. Lung. 1983;161(4):229-234.

34. Gilfillan AM, Smart DA, Rooney SA. Comparison of the enzyme activities of phosphatidylcholine, phosphatidylglycerol and phosphatidylinositol synthesis in freshly isolated type II pneumocytes and whole lung from the adult rat. Biochim Biophys Acta. 1986;877(1):151-157.

35. Ballard PL. Hormones and lung maturation. Monogr Endocrinol. 1986;28:1-354.

36. Ikegami M, Jobe AH, Yamada T, Seidner S. Relationship between alveolar saturated phosphatidylcholine pool sizes and compliance of preterm rabbit lungs. The effect of maternal corticosteroid treatment. Am Rev Respir Dis. 1989;139(2):367-369.

37. Clements JA and Tooley WH. Kinetics of surfaceactive material in the fetal lung. In: Hodson WA, ed. Development of the Lung. W.A. New York, New York: Marcel Dekker; 1977:349-366.

38. Soupene E, Fyrst H, Kuypers FA. Mammalian acyl-CoA:lysophosphatidylcholine acyltransferase enzymes. Proc Natl Acad Sci U S A. 2008;105(1):88-93.
39. Hishikawa D, Shindou H, Kobayashi S, Nakanishi H, Taguchi R, Shimizu T. Discovery of a lysophospholipid acyltransferase family essential for membrane asymmetry and diversity. Proc Natl Acad Sci US A. 2008;105(8):2830-2835.

40. Shindou $\mathrm{H}$, et al. A single enzyme catalyzes both platelet-activating factor production and membrane biogenesis of inflammatory cells. Cloning and characterization of acetyl-CoA:LYSO-PAF acetyltransferase. J Biol Chem. 2007;282(9):6532-6539.

41. Jain S, Zhang X, Khandelwal PJ, Saunders AJ, Cummings BS, Oelkers P. Characterization of human lysophospholipid acyltransferase 3. J Lipid Res. 2009; 50(8):1563-1570.

42. Kazachkov M, Chen Q, Wang L, Zou J. Substrate preferences of a lysophosphatidylcholine acyltransferase highlight its role in phospholipid remodeling. Lipids. 2008;43(10):895-902.

43. Cao J, et al. Molecular identification of a novel mammalian brain isoform of acyl-CoA:lysophospholipid acyltransferase with prominent ethanolamine lysophospholipid acylating activity, LPEAT2 J Biol Chem. 2008;283(27):19049-19057.

44. van Meer G, Voelker DR, Feigenson GW. Membrane lipids: where they are and how they behave. Nat Rev Mol Cell Biol. 2008;9(2):112-124.

45. Wang L, Magdaleno S, Tabas I, Jackowski S. Early embryonic lethality in mice with targeted deletion of the CTP:phosphocholine cytidylyltransferase alpha gene (Pcyt1a). Mol Cell Biol. 2005;25(8):3357-3363.

46. Tian Y, Zhou R, Rehg JE, Jackowski S. Role of phosphocholine cytidylyltransferase alpha in lung development. Mol Cell Biol. 2007;27(3):975-982.

47. Chakraborti S. Phospholipase A(2) isoforms: a perspective. Cell Signal. 2003;15(7):637-665.

48. Dennis EA. Diversity of group types, regulation, and function of phospholipase A2. J Biol Chem. 1994; 269(18):13057-13060.

49. Nijssen JG, van den Bosch H. Cytosol-stimulated remodeling of phosphatidylcholine in rat lung microsomes. Biochim Biophys Acta. 1986;875(3):450-457.

50. Garcia A, Newkirk JD, Mavis RD. Lung surfactant synthesis: a Ca++-dependent microsomal phospholipase A2 in the lung. Biochem Biophys Res Commun. 1975;64(1):128-135.

51. Longmore WJ, Oldenborg V, van Golde LM. Phospholipase A2 in rat-lung microsomes: substrate specificity towards endogenous phosphatidylcholines. Biochim Biophys Acta. 1979;572(3):452-460.

52. Heath MF, Jacobson W. The action of lung lysosomal phospholipases on dipalmitoyl phosphatidylcholine and its significance for the synthesis of pulmonary surfactant. Pediatr Res. 1980;14(3):254-258.

53. Fisher AB, Dodia C. Role of phospholipase A2 enzymes in degradation of dipalmitoylphosphatidylcholine by granular pneumocytes. J Lipid Res. 1996; 37(5):1057-1064.

54. Fisher AB, Feinstein SI, Kim TS, Dodia C, Skach W. Identification of a cDNA for $\mathrm{Ca}++-$ independent phospholipase A2 of lung lamellar bodies/lysosomes. Chest. 1997;111(6 Suppl):88S-89S.

55 . Heath MF, Jacobson W. The nature of the phospholipases A of lung lamellar bodies. Pediatr Res. 1980; 14(6):846-847.

56. Heath MF, Jacobson W. Phospholipases A1 and A2 in lamellar inclusion bodies of the alveolar epithelium of rabbit lung. Biochim Biophys Acta. 1976; 441(3):443-452

57. Kim TS, et al. Identification of a human cDNA clone for lysosomal type Ca2+-independent phospholipase A2 and properties of the expressed protein. J Biol Chem. 1997;272(4):2542-2550.

58. Mo Y, et al. 1-Cys peroxiredoxin knock-out mice express mRNA but not protein for a highly related intronless gene. FEBS Lett. 2003;555(2):192-198.

59. Fisher AB, Dodia C, Feinstein SI, Ho YS. Altered lung phospholipid metabolism in mice with targeted deletion of lysosomal-type phospholipase A2. J Lipid Res. 2005;46(6):1248-1256.

60. Ikegami M, Whitsett JA, Martis PC, Weaver TE. Reversibility of lung inflammation caused by SP-B deficiency. Am J Physiol Lung Cell Mol Physiol. 2005; 289(6):L962-L970.

61. Maruscak A, Lewis JF. Exogenous surfactant therapy for ARDS. Expert Opin Investig Drugs. 2006; 15(1):47-58.

62. Spragg RG, et al. Effect of recombinant surfactant protein C-based surfactant on the acute respiratory distress syndrome. NEngl J Med. 2004;351(9):884-892.

63. Spragg R. Surfactant for acute lung injury. Am J Respir Cell Mol Biol. 2007;37(4):377-378.

64. Pettenazzo A, Ikegami M, Seidner S, Jobe A. Clearance of surfactant phosphatidylcholine from adult rabbit lungs. J Appl Physiol. 1988;64(1):120-127.

65. Mason RJ, et al. Keratinocyte growth factor and the transcription factors C/EBP alpha, C/EBP delta, and SREBP-1c regulate fatty acid synthesis in alveolar type II cells. J Clin Invest. 2003;112(2):244-255.

66. Schuberth C, Buchberger A. Membrane-bound Ubx 2 recruits $\mathrm{Cdc} 48$ to ubiquitin ligases and their substrates to ensure efficient ER-associated protein degradation. Nat Cell Biol. 2005;7(10):999-1006.

67. Batenburg JJ, den Breejen JN, Yost RW, Haagsman HP, van Golde LM. Glycerol 3-phosphate acylation in microsomes of type II cells isolated from adult rat lung. Biochim Biophys Acta. 1986;878(3):301-309.

68. Bridges JP, Wert SE, Nogee LM, Weaver TE. Expression of a human surfactant protein $\mathrm{C}$ mutation associated with interstitial lung disease disrupts lung development in transgenic mice. J Biol Chem. 2003; 278(52):52739-52746.

69. Rice WR, Conkright JJ, Na CL, Ikegami M, Shannon JM, Weaver TE. Maintenance of the mouse type II cell phenotype in vitro. Am J Physiol Lung Cell Mol Physiol. 2002;283(2):L256-L264.

70. Bligh EG, Dyer WJ. A rapid method of total lipid extraction and purification. Can J Biochem Physiol. 1959;37(8):911-917.

71. Mason RJ, Nellenbogen J, Clements JA. Isolation of disaturated phosphatidylcholine with osmium tetroxide. J Lipid Res. 1976;17(3):281-284.

72. Cesarone CF, Bolognesi C, Santi L. Improved microfluorometric DNA determination in biological material using 33258 Hoechst. Anal Biochem. 1979; 100(1):188-197.

73. Ikegami M, Whitsett JA, Jobe A, Ross G, Fisher J, Korfhagen T. Surfactant metabolism in SP-D genetargeted mice. Am J Physiol Lung Cell Mol Physiol. 2000;279(3):L468-L476.

74. Bartlett GR. Phosphorus assay in column chromatography. J Biol Chem. 1959;234(3):466-468.

75. Schoel WM, Schurch S, Goerke J. The captive bubble method for the evaluation of pulmonary surfactant: surface tension, area, and volume calculations. Biochim Biophys Acta. 1994;1200(3):281-290.

76. Akei $\mathrm{H}$, et al. Surface tension influences cell shape and phagocytosis in alveolar macrophages. Am J Physiol Lung Cell Mol Physiol. 2006;291(4):L572-L579. 